\title{
Rich Club organization supports a diverse set of functional network configurations
}

\section{Authors}

Mario Senden ${ }^{1}$, Gustavo Deco ${ }^{2,3}$, Marcel A. de Reus ${ }^{5}$, Rainer Goebel ${ }^{1,4}$, and Martijn van den Heuvel $^{5}$

1) Department of Cognitive Neuroscience, Faculty of Psychology and Neuroscience, Maastricht University, 6201BC Maastricht, The Netherlands

2) Center for Brain and Cognition, Computational Neuroscience Group, Department of Information and Communication Technologies, Universitat Pompeu Fabra, Roc Boronat 138, Barcelona, 08018, Spain

3) Institució Catalana de la Recerca i Estudis Avançats (ICREA), Universitat Pompeu Fabra, Passeig Lluís Companys 23, Barcelona, 08010, Spain

4) Department of Neuroimaging and Neuromodeling, Netherlands Institute for Neuroscience, an Institute of the Royal Netherlands Academy of Arts and Sciences (KNAW), 1105BA Amsterdam, The Netherlands

5) Brain Center Rudolf Magnus, Department of Psychiatry, University Medical Center Utrecht, Brain Center Rudolf Magnus, 3508 GA Utrecht, The Netherlands

\section{Address for Correspondence}

Mario Senden, Department of Cognitive Neuroscience, Faculty of Psychology and

Neuroscience, Maastricht University, Oxfordlaan 55, 6200 MD Maastricht, P.O. Box 616,

The Netherlands, Phone number: +31 4338 82071;

Email: mario.senden@maastrichtuniversity.nl 


\section{Abstract}

Brain function relies on the flexible integration of a diverse set of segregated cortical modules, with the structural connectivity of the brain being a fundamentally important factor in shaping the brain's functional dynamics. Following up on macroscopic studies showing the existence of centrally connected nodes in the mammalian brain, combined with the notion that these putative brain hubs may form a dense interconnected 'rich club' collective, we hypothesized that brain connectivity might involve a rich club type of architecture to promote a repertoire of different and flexibly accessible brain functions. With the rich club suggested to play an important role in global brain communication, examining the effects of a rich club organization on the functional repertoire of physical systems in general, and the brain in particular, is of keen interest. Here we elucidate these effects using a spin glass model of neural networks for simulating stable configurations of cortical activity. Using simulations, we show that the presence of a rich club increases the set of attractors and hence the diversity of the functional repertoire over and above the effects produced by scale free type topology alone. Within the networks' overall functional repertoire rich nodes are shown to be important for enabling a high level of dynamic integrations of low-degree nodes to form functional networks. This suggests that the rich club serves as an important backbone for numerous coactivation patterns among peripheral nodes of the network. In addition, applying the spin glass model to empirical anatomical data of the human brain, we show that the positive effects on the functional repertoire attributed to the rich club phenomenon can be observed for the brain as well. We conclude that a rich club organization in network architectures may be crucial for the facilitation and integration of a diverse number of segregated functions.

Key words: rich club, functional repertoire, connectivity, spin glass model, connectome 


\section{Introduction}

The human brain is composed of a large set of anatomically distinct regions and local clusters indicative of segregated neural information processing. The execution of higher order cognitive functions such as memory, perception, and attention demands the integration of this information via distributed computation. Computationally driven theories of cognition hypothesize that the brain may achieve integration of subsystems by flexibly arranging cortical areas into temporal functional networks in accordance with goal-related requirements (Baars, 2005; Ghosh et al. 2008; Deco et al. 2010). The exact nature as well as the size of the set of possible functional network configurations, referred to as the brain's functional repertoire, has been suggested to relate directly to the structural architecture of the brain (Deco et al. 2010; Deco et al. 2012; Senden et al. 2012). Network architectures that involve a scale free topology; meaning that the degree distribution follows a power law function indicating the existence of a small number of high-degree nodes, have been shown to be able to display a particularly diverse number of functional configurations (Deco et al. 2012).

In addition to a heavy tailed degree distribution the human brain has been shown to contain hubs which are not only individually 'rich' in connectivity but additionally show a dense level of interconnectivity (Colizza et al. 2006; Van den Heuvel \& Sporns, 2011). This collective of highly interconnected hubs has been termed the 'rich club' analogously to the organization of social systems in which individuals rich in connections tend to form strongly interconnected clubs, taking a central position in the overall system (McAuley et al. 2007; Zhou \& Mondragon, 2004). Similarly, neural rich clubs have been hypothesized to act as a central high-capacity backbone for global communication (Van den Heuvel et al. 2012) and integration (Van den Heuvel and Sporns, 2013) in the brain.

In this computational study we hypothesized that the presence of a rich club in an otherwise scale free type architecture further expands the functional repertoire of a system. To 
test this hypothesis a steady-state attractor model was applied to artificial network architectures as well as human experimental anatomical data to examine the influence of a network's architecture on its functional diversity. In addition, we studied the shape of the functional repertoire with a special focus on the comparison between scale free architectures that show rich club organization versus scale free architectures without rich club organization. Overall, our findings suggest that the presence of a central rich club on top of a scale free architecture may lead to an additional gain in the diversity of a network's functional repertoire, suggesting that a structural rich club in neural systems may further expand the brain's functional diversity. Interestingly, our findings further indicate that the presence of a rich club enhances the capability of the system to functionally integrate areas of low structural degree.

\section{Methods}

\section{Artificial Network Architectures}

Six types of network architectures were examined (figure 1): Regular (REG), Random (RAND), Small world (SW), Barabasi-Albert scale free including a rich club formation (SF$\mathrm{RC})$, Scale free without rich club formation (SF), and Scale free whose rich nodes are secluded from one another (SF-negRC). Each network contained exactly 24 nodes with each node (results of networks of $\mathrm{N}=30$ nodes are presented in the supplemental materials), on average, making four bidirectional, unweighted connections to other nodes, resulting in a total of 96 connections (also referred to as edges) per network. Small networks were chosen due to the high computational demand of the spin glass model (see supplemental information). In what follows, the formation of these networks are described, starting with the REG, RAND and SW class, followed by the three categories of SF networks.

Regular network (REG). A single regular network was generated by ordering 24 nodes on a circular lattice and subsequently connecting each node to its two nearest neighbors on both 
sides. Random networks (RAND). A set of 100 random networks were generated using the algorithm described by Watts and Strogatz (1998) which rewires each connection in a regular network with a prefixed probability $p$. For random networks the probability of rewiring was set equal to one.

Small world networks (SW). A set of 100 small world networks were generated using the rewiring algorithm described by Watts and Strogatz (1998) with a probability of rewiring set to 0.25 , resulting in a network with a small world topology in which the majority of edges are between neighboring nodes, with a few connections forming short-cut connections between remote parts of the network.

Scale free networks. Scale free networks were generated by applying the Barabasi-Albert algorithm (Barabási \& Albert, 1999) on random seed networks. This algorithm employs the principle of preferential attachment in which the probability that a newly added node will form a connection with an existing node is proportional to the degree of the existing node. As a result, the subset of seed nodes will end up as the most densely connected nodes (i.e. hubs) in the generated network. Three types of scale free networks were formed:

1. Scale free networks without a rich club $(S F)$. A set of 100 scale free networks containing no rich club (SF) were generated by performing the Barabasi-Albert algorithm (Barabási \& Albert, 1999) on a seed of intermediate density. The seed set had nine nodes each making four connections leading to the formation of a set of scale free networks in which the starting nodes show a high level of connectivity (i.e. form hubs) but no central rich club.

2. Scale free networks with a rich club $(S F-R C)$. A set of 100 scale free networks containing a dense rich club (SF-RC) were generated by performing the Barabasi-Albert algorithm (Barabási \& Albert, 1999) on a dense seed set of six nodes each making four connections, leading to the formation of a set of scale free networks in which the starting nodes show a high level of connectivity (i.e. form hubs) as well as a dense level of interconnectivity forming a central rich club. 
3. Scale free networks with a negative rich club. A set of 100 scale free networks and with a negative rich club (SF-negRC) was generated by performing the Barabasi-Albert algorithm (Barabási \& Albert, 1999) using a sparse seed of twelve nodes with each making four connections, resulting in a set of networks with a topology that showed a scale free degree distribution, but in which the hubs had a below chance level of inter-connectivity (i.e. formed a below chance level of connectivity).

\section{Spin Glass Model}

To examine the link between network architecture and functional entropy we adopted the analytically solvable Ising spin glass model from Deco et al. (2012). The model, which is isomorphic to the discrete Hopfield net (Hopfield, 1982), studies the characteristics of the attractor landscapes emerging in a spin glass neural model. The spin glass model includes a network of spins which can be in one of two possible states $(0,1)$. The spins are symmetrically coupled according to a set of connections (here, the undirected binary connections are edges given by an underlying structural network and the spins represent cortical areas), allowing for interactions among them. The state of a spin $i$ is given by $S_{i}, C$ denotes the adjacency matrix associated with the network architecture under consideration. The probability of finding the network in a specific global configuration $S$ (indicated by a superindex $\alpha$ ) is derived from the Boltzmann-Gibbs distribution (Gibbs, 1905) ${ }^{1}$ :

$$
P^{\alpha}=\frac{e^{-\varepsilon H^{\alpha}}}{Z}
$$

where $\varepsilon$ is the reciprocal of the thermodynamic temperature of the system (here, $\varepsilon=1$ ) and $Z$ is the partition function

\footnotetext{
${ }^{1}$ A more recent English description is given in (Landau \& Lifs $\square$ ic, 2007)
} 
$Z=\sum_{\alpha} e^{-\varepsilon H^{\alpha}}$

The energy function $\mathrm{H}^{\alpha}$ is given by

$H^{\alpha}=\frac{1}{2} \theta \Sigma_{i} S_{i}^{\alpha}-\frac{1}{2} W \Sigma_{i, j} C_{i j} S_{i}^{\alpha} S_{j}^{\alpha}$

with $\theta$ being the transition threshold at which a spin changes its state and $W$ a scaling parameter for the adjacency matrix $C$ referred to as global coupling strength. Here, $\theta$ was set to 12 . The entropy of the system is given by:

$E=-\Sigma_{\alpha} P^{\alpha} \log P^{\alpha}=\frac{\Sigma_{\alpha} \varepsilon H^{\alpha} e^{-\varepsilon H^{\alpha}}}{Z}+\log Z$

and reflects the number of accessible states of the system and serves to describe the attractor landscape of the system. Note that the maximal entropy that a system with $N$ binary nodes can exhibit is given by:

$\max \left(E_{N}\right)=\log \left(2^{N}\right)$

Solving the spin glass model, which requires examination of $2^{\mathrm{N}}$ states, is computationally demanding even for small networks (see supplementary figure 1). In order to investigate spin glass models with up to $2^{30}$ states the energy function as well as the summands of the partition function were calculated for all states in parallel on graphical processing units (GPUs). To be able to investigate spin glass models with more than $\mathrm{N}=30$ nodes and $2^{30}$ states we adopted a Metropolis-Hastings Algorithm (Hastings, 1970; 
Metropolis, Rosenbluth, Rosenbluth, Teller, \& Teller, 1953), sampling a subset out of the total $2^{\mathrm{N}}$ solutions (see Appendix for details).

We additionally derived the mutual information from the probability distribution $\mathrm{P}^{\alpha}$ across states, which allows for calculation of both joint and marginal probabilities. Specifically, the mutual information $M$ between nodes $i$ and $j$ is given by:

$M_{i, j}=\sum_{S_{i}=0}^{1} \sum_{S_{j}=0}^{1} P\left(S_{i}, S_{j}\right) \log \left(\frac{P\left(S_{i}, S_{j}\right)}{P\left(S_{i}\right) P\left(S_{j}\right)}\right)$

Where $P\left(S_{i}\right)$ and $P\left(S_{j}\right)$ are the marginal probabilities of nodes $i$ and $j$, respectively, and $P\left(S_{i}, S_{j}\right)$ are the joint probabilities of the two nodes.

\section{Degree-Related Connectivity}

Degree-related connectivity (DRC) was used to quantify the strength with which nodes of a certain degree range are interlinked. More formal, it measures the total observed connectivity between nodes of degree $k$ and nodes of degree $l$ divided by the number of possible structural connections between those nodes, with $k$ and $l$ thus being structural degrees obtained from the network architecture.

$D R C_{k, l}=\frac{\sum_{i \in N_{k}} \sum_{j \in N_{l}} A(i, j)}{\sum_{i \in N_{k}} i \sum_{j \in N_{l}} j}$

where $i$ and $j$ are nodes from the sets $N_{k}$ and $N_{l}$ comprising all nodes of degree $k$ and $l$ within the underlying adjacency matrix, respectively and $A$ is the connectivity under consideration. In the case that nodes of equal degree are considered (i.e. $k$ is equal to $l$ ) both $i$ and $j$ are drawn from the same population $N_{k l}$. Here, potential self-connections are omitted by drawing $j$ from $N_{k l}$ and $i$ from $N_{k l} l\{j\}$. For structural DRC $A$ is identical to the adjacency matrix $C$. To 
compute functional DRC (i.e. DRC based on functional connectivity) $A$ was identical to a functional connectivity matrix given by the mutual information matrix $M I$ (or by the crosscorrelation matrix for empirical data). Division by the number of possible structural connections between nodes of certain degrees is necessary since certain degrees can be overrepresented in network architectures and hence bias the DRC. This is especially true for scale free architectures where low-degree nodes are overabundant.

\section{Human Empirical Data}

In addition to the created artificial network architectures (i.e. regular, random, small world, and three types of scale free networks of 24 nodes) we investigated the behavior of the spin model on empirical human anatomical connectivity data, derived from diffusion weighted imaging. Group-averaged structural data and information on the brain's functional connectivity architecture were acquired on the basis of diffusion weighted MRI and restingstate fMRI recordings, respectively, based on data described in the recent paper of Collin et al. (2013b). In short (we refer to Collin et al (2013b) for a detailed description of the acquisition and analysis procedure), the selected subset described the reconstructed anatomical connections between 68 areas of the cortex of 23 healthy adult subjects derived from deterministic streamline tractography. Next, a binary group-average structural connectivity matrix was formed by including all connections that were found to be present in at least 70 percent of the participants (de Reus and Van den Heuvel 2013). In parallel, a group-averaged functional connectivity matrix was formed by averaging the individual functional connectivity matrices over the group of subjects, describing the level of correlation between the resting-state time-series of each pair of the included 68 cortical regions.

Structural DRC was calculated for empirical structural connectivity matrices while functional DRC was calculated for empirical functional connectivity matrices. All empirical connectivity matrices comprised 68 nodes (i.e. both hemispheres). Computation of empirical DRC was 
performed in a similar fashion as computed for the artificial networks. However, computation of functional DRC was based on the group-averaged functional correlation matrix (68 nodes); taking into account information on the positive correlations (i.e. negative correlations were ignored). The procedure to calculate functional DRC remained otherwise identical to the one used for $M I$ in the artificial networks.

\section{Results}

\section{Rich club organization}

The three categories of scale free networks were quantitatively distinguished by their normalized rich club coefficients given by the fraction of the actual number of connections between nodes with degree larger than $k$ to the maximal number of connections among these nodes (Colizza et al., 2006; Zhou \& Mondragon, 2004). Rich club coefficients were calculated for each of the scale free networks and normalized to a set of random networks with equal degree distributions with a normalized rich club coefficient exceeding 1 signifying the presence of rich club organization within a network (Colizza et al., 2006; Van den Heuvel \& Sporns, 2011; Van den Heuvel \& Sporns, 2013). Figure 2 shows the rich club coefficients for the three classes of scale free networks. Validating our network generation models, the SF-RC class revealed an average normalized rich club function that exceeds 1 in the interval [4,7] (dark blue line), the SF class (blue line) showed an average rich club coefficient close to 1, and the SF-negRC class (light blue) showed an average rich club coefficient below 1.

\section{Entropy}

The entropy of each of the network models was examined by using a spin glass model in which spins are arranged on a lattice according to an underlying structural architecture and connection strengths are systematically increased by a global coupling factor. At critical coupling the system as a whole becomes multistable and exhibits a set of attractors 
representing the functional repertoire of the system, with the system's level of entropy reflecting the size of the attractor set (Deco et al. 2012). Figure 3 shows the entropy of the attractors for the set of networks as a function of the global coupling strength for all classes of artificial networks. Supporting previous findings (Deco et al. 2012), the entropy obtained across a range of global coupling strength was found to be higher for the scale free network architectures as compared to SW, RAND, and REG (for an analysis with networks comprising 30 nodes see supplementary figures 2 and 3). However, importantly, being the focus of our study, within the class of scale free architectures, networks of the SF-RC class

(i.e. containing a rich club) revealed a higher level of entropy (mean entropy $\mu \mathrm{E}=10.23$ ) than networks of the $\mathrm{SF}(\mu \mathrm{E}=9.54)$ and SF-negRC $(\mu \mathrm{E}=8.79)$ class $[\mathrm{p}<.001$ in an ANOVA test with entropy at the critical coupling point $(W=3)$ as dependent variable and rich club (rich club, no rich club, negative rich club) as fixed factor]. The largest theoretically possible entropy among network architectures comprising 24 nodes was computed as $\max \left(\mathrm{E}_{24}\right)=$ 16.636.

\section{Functional Repertoire}

The marginal probabilities of nodes being active, i.e. their corresponding spins being in an 'up' state, give an indication as to how many nodes are active at any moment which limits the number of possible configurations. The largest number of possible configurations can be achieved by systems with $50 \%$ of their nodes active for any given configuration. Interestingly, the scale free network architectures considered here approached the 50\% mark. In detail, for SF network architectures the most common global configurations were those where 65.5 percent of the nodes were active. However, for the SF-RC network architectures the most common global configurations were those where 58.3 percent of the nodes were active, higher (and therefore more diverse) as compared to the SF networks. Finally, for SF-negRC network architectures the most common global configurations were those where 66.7 percent of the 
nodes were active. For comparison, for regular, random, and small world network architectures the most common global configurations were those where, either all or none, 79.2 percent, and 70.8 percent, of the nodes were active, respectively.

While these numbers represent the overall number of nodes being active for the most configurations, the distribution across nodes is not uniform. Specifically, high-degree nodes in scale free network architectures revealed a particularly high probability of being active in such a way that their spins were in an 'up' state for all of the common global configurations. At the same time low-degree nodes in these network architectures were active for $50 \%$ or less of the common global configurations. This is illustrated in figure 4 where the marginal probability of being active is shown for each of the 24 nodes within a network architecture with nodes sorted ascending by their degree (random network architectures served as a baseline). Rich nodes (i.e. the set of high degree nodes) revealed to be active almost unequivocally, whereas low degree nodes were far freer to switch between 'up' and 'down' states. Although this effect was present for all scale free type architectures, it was found to be the most pronounced for SF-RC architectures.

The high degree - low degree subdivision of the functional repertoire was further examined using degree related connectivity. Figure 5a and b show the average structural and functional DRCs over the 100 matrices of SF, SF-RC and SF-negRC architectures, with Figure 5c showing the Euclidean distance between the centers of mass of functional and structural DRC distributions. The structural connectivity for SF network architectures largely featured connections among nodes of moderate degree with the mean center of mass (which in symmetric distributions such as these lies along the diagonal) over all structural DRCs at $\mu \mathrm{k}=6.73$ [6.62 6.83]. As expected from our manipulations, for SF-RC the mean center of mass over all structural DRCs shifted toward higher degrees $\mu \mathrm{k}=7.19$ [7.08 7.3]. For SFnegRC the mean center of mass over all structural DRCs was located at $\mu \mathrm{k}=6.29$ [6.19 6.39]. With regard to functional DRC, low degree functional connections were found to be 
prominent for SF-RC network architectures but less prominent in SF and SF-negRC network architectures as the mean centers of mass were located at $\mu \mathrm{k}=5.25$ [5.14 5.35], $\mu \mathrm{k}=6.15$ [6.04 6.25], and $\mu \mathrm{k}=6.21$ [6.12 6.3], respectively. These differences among center of mass for functional DRCs were found to be significant in an ANOVA test with $\mathrm{p}<.001$. Subsequent pairwise comparison revealed that the center of mass for SF-RC was located at significantly lower degree nodes as compared to SF $(\mathrm{p}<.001)$ and SF-negRC $(\mathrm{p}<.001)$, whereas no significant difference was observed between the centers of mass of the latter two $(p=.34)$. Taken together, these findings suggests that while SF and SF-negRC mainly recruit moderate nodes for the formation of functional networks, a SF-RC architecture may enable the formation of a diverse set (and overall, a more diverse set as reflected by the higher level of entropy, figure 3) of functional configurations recruiting low-degree nodes. Since low-degree nodes are most prominent in scale free network architectures (see supplementary figure 4 for details) their integration allows for a particularly diverse functional repertoire.

The third column of figure 5 shows Euclidean distances between centers of mass for functional and structural DRC, showing that functional interactions can be strong in the absence of structural connections. The distribution of Euclidean distances was obtained by a bootstrapping procedure sampling the structural and functional centers of mass from the 100 networks considered for each class of network architectures. Figure 5c emphasizes that SFRC networks show strong connectivity among low-degree nodes even though structural connections were mostly centered around moderate to high-degree nodes. For SF and SFnegRC network architectures the formation of functional connections was found to be more limited to nodes which already share anatomical connections.

\section{Results on empirical human connectivity}

Employing the Metropolis-Hastings algorithm (Hastings, 1970; Metropolis, Rosenbluth, Rosenbluth, Teller, \& Teller, 1953) we examined the (estimated) entropy exhibited by a spin 
glass model whose underlying connectivity matrix was a binary empirical structural connectivity matrix of the human brain (HUM) comprising 68 nodes derived from diffusion MRI data ${ }^{2}$. Additionally, the results were contrasted with those obtained for randomly rewired versions (HUM-RW) of the human connectivity matrix leaving the degree distribution intact but removing the rich club phenomenon as well as with artificial regular, random, and small world connectivity matrices of the same order and sparsity (Maslov and Sneppen 2002). Figure 6 shows the entropy of the attractors for the set of networks comprising 68 nodes as a function of the global coupling strength for all classes of network architectures. Consistent with the simulations of network architectures comprising 24 nodes, the entropy obtained across a range of global coupling strengths was found to be higher for human structural connectivity as compared to small world, random, and regular network architectures. Furthermore, human structural connectivity (containing a rich club structure) revealed a higher level of entropy (mean entropy $\mu \mathrm{E}=8.64$ ) than rewired versions lacking a rich club $(\mu \mathrm{E}=7.97)[\mathrm{p}<.001$ in a t-test with entropy at the critical coupling point $(W=1)$ as dependent variable].

We additionally examined the DRC obtained from empirical human structural and functional connectivity. We performed this analysis on functional connectivity as obtained from resting state fMRI. Figure 7 shows the empirical structural (a) and empirical functional DRC (b). As can be seen in figure $7 b$, functional clusters among low $(k=[4,10])$ degree nodes are prominent. Since these nodes together constitute $46 \%$ of all nodes it is their integration that leads to a diverse functional repertoire. While moderate-degree nodes share structural as well as functional connections (figure 6a and b), functional coupling among low-degree nodes

\footnotetext{
${ }^{2}$ We investigated the suitability of our implementation of the Metropolis-Hastings algorithm by obtaining the entropy of matrices comprising 30 nodes both analytically as well as using the algorithm. See supplementary figures 2 and 3 .
} 
must occur in the absence of direct structural connections. This observation overlapped with the simulated results of the artificial network architectures ( $n=24$ nodes, figure $5 c)$.

\section{Discussion}

Our findings demonstrate that the presence of a central rich club core of mutually densely connected hub nodes constitutes a benefit to a network's functional repertoire. Across random, regular, small world, and three types of scale free networks as well as human empirical structural connectivity, the number of attractors (i.e. entropy) in the spin glass model was found to be largest for scale free networks displaying a rich club organization and hence higher than scale free networks that lacked dense connectivity between high degree hubs (i.e. SF and SF-nonRC, figure 3).

At the level of critical coupling, the spin glass model has been shown to exhibit a number of unique attractors representing the diversity of the system's functional repertoire (Deco et al. 2012). It has previously been suggested that a scale free architecture might be beneficial for a network with respect to obtaining a high level of entropy and thus a more diverse functional repertoire as compared to systems with an underlying regular, small world or random architecture (Deco et al. 2012). Extending these findings, our results now show that the presence of a central rich club within otherwise scale free architectures leads to an additional increase in the number of unique functional networks the system can sustain.

In context of studies suggesting that higher numbers of attractors are linked to a more diverse repertoire of functional dynamics (Deco et al. 2012), our current computational findings may add to our understanding of rich club formation in neural systems. In the mammalian brain, and likely neural systems in general, the rich club has been suggested to form a high-capacity backbone allowing for dynamic routing of information (Collin et al. 2013; Harriger et al. 2012; Towlson et al. 2013; Van den Heuvel et al. 2012; Zamora-Lopez et al. 2011). In this 
context, we interpret our current findings as evidence for the presence of a structural central rich club in the mammalian brain to have a positive effect on the diversity of the brain's functional repertoire and to permit functionally segregated cortical areas to become integrated when needed (Zamora-Lopez et al 2011). Indeed, our first examination of applying the spin glass model to simulate neural connectivity on the empirical anatomical connectivity data of the human brain suggests that the rich club may promote functional diversity specifically by supporting integration of information between low-degree nodes. As such, these simulation findings tend to suggest that a structural rich club may enable the dynamic formation of functional networks in which information can be integrated specifically for the performance of a given task (Bassett et al., 2009; Van den Heuvel and Sporns 2013a). Such an observation runs in parallel with modern theories of cognition hypothesizing the existence of a 'global workspace' in which segregated communities can exchange information through means of neural interaction (Baars, 2005). With regard to the shape of the functional repertoires, our results indeed suggest that the presence of a rich club enables the flexible coordination of low-degree nodes into unique functional networks. That is, the functional repertoire appears to feature a universally involved backbone in the form of almost constantly active rich nodes enabling and sustaining specific (re-)configurations among low-degree nodes. In doing so, rich nodes would naturally participate in many functional networks of the brain, which has indeed recently been suggested (Van den Heuvel and Sporns 2013a; de Reus and Van den Heuvel 2013). These findings are further in line with a previous study by Braga et al. (2013) which revealed that densely connected cortical areas carry functional traces of many functionally specific functional networks. This suggests that an anatomical rich club enables configurations of functional networks by sustaining activity of low degree nodes as well as by routing information among them. Our and related findings thus converge to the notion of a rich club to be beneficial for ensuring both a high level of functional diversity as well as 
flexible integration and exchange of information among low-degree nodes (Van den Heuvel and Sporns 2013a).

Some points need to be considered when interpreting the findings of our study. First, the artificial network architectures studied semi-analytically are comparatively small, relating to the high computational cost of the investigation of the spin glass model in which every possible spin configuration is considered in detail (see supplemental materials). However, the study of Deco et al. (2012) showed that while network size affects absolute levels of entropy, the relative effect that different network architectures have on entropy was shown not to be affected by size. Indeed, a similar effect was observed when comparing our $\mathrm{N}=24$ and $\mathrm{N}=30$ network results, as well as by the approximations of global entropy for human empirical data showing that the effect related to the rich club phenomenon is also present in biologically relevant networks of larger order.

Second, we note that our spin glass simulation results do not necessarily provide information on the level of flow of information between low degree and/or high degree nodes: the spin glass model as applied in our study can only convey information as to the activation state (i.e. 'up' or 'down') of nodes. That is, the functional DRC in our simulations only reflects in how far nodes of a certain degree range can form dynamic (re-)configurations of activation states and does not reveal in how far (re-)configurations of actual information flow occur. Our simulation results can therefore only to a certain extent be compared to empirical functional DRC observations derived from correlations between time-series. Future studies in this area might allow for more detailed investigation of temporal dynamics and might reveal whether activity fluctuation ride on top of a global high activation envelope (i.e. the up states), hence providing more information on the dynamical character of functional and effective connectivity. Nevertheless, co-activation is likely a necessary prerequisite for information flow between brain regions and the configurations of co-activation patterns 
provide a constraint on the patterns of such information flow. Additionally, our simulation results of a constantly active rich club and low functional DRC between rich club nodes are in agreement with recent empirical findings of high metabolic demand of high degree cortical areas (Bullmore and Sporns 2012) and rich club nodes in particular and with observations of relatively low functional connectivity among rich club nodes as measured during resting state (Collin et al. 2013b). Rich club organization of brain networks has been suggested to potentially reflect the capacity of a neural system to facilitate neural interplay and neural communication among high degree hub regions when integration of information between low-degree segregated functional domains is needed (Braga et al. 2013; Van den Heuvel and Sporns 2013a, 2013b; de Reus and Van den Heuvel 2013), a hypothesis consistent with our simulation results suggesting that a rich club can increase the overall capacity of the network to boost functional connectivity between low degree nodes. Future studies examining the effects of rich club organization in more detail using neural mass models which allow for the simulation of neural time-series and thus simulated functional couplings between regions would be of high interest to further elucidate the role of rich club organization to functional network organization and integration.

Third, the comparability of functional connectivity measured by mutual information in the spin glass model to the commonly used correlation metric for empirical resting-state fMRI measurements is debatable. Nonetheless, results stemming from both were highly consistent. In our paper we advocate that our findings indicate that functional integration of low-degree nodes is promoted by scale free systems that show rich club organization, including the (human) brain. However, our computational results should be interpreted largely as an attempt to provide a theoretical framework to be used for more in depth empirical as well as simulation research than we can provide here (Breakspear et al. 2010; Friston \& Dolan, 2010). A particularly interesting prediction of our results is that task related functional networks should be distinguishable mainly by specific co-activations of low degree cortical 
areas with the rich club being present and active across multiple task related functional networks. Another interesting topic of investigation for future studies would be the simulation of effects of disrupted anatomical rich club organization on the functional repertoire. Such studies might for example provide more insight into the functional effects of abnormal rich club formation as observed in patients with schizophrenia (Van den Heuvel et al. 2013; Collins et al. 2011; Collin et al. 2013a; Yu et al., 2012).

In conclusion, our study provides computational support for the notion of rich club organization aiding the brain's overall repertoire of functional diversity (Zamora-Lopez 2011; Van den Heuvel and Sporns 2013). Networks with a scale free architecture combined with the presence of a central densely connected rich club revealed a higher level of entropy compared to networks with a random, regular, small world and indeed other scale free architectures. Additionally, our findings tend to suggest that networks featuring rich club organization allow for the functional integration of specialized cortical regions of low macroscopic degree. As such, our findings provide evidence in support of the notion that a rich club in neural systems may form a neural substrate that both enriches as well as modulates the brain's repertoire of distinct specialized brain functions to allow for the flexible integration of cortical areas into functional networks. Future research, investigating how additional graph theoretical properties affect the brain's functional repertoire, how they interact with a rich club, and how simulated and empirical disease related damage to the rich club might reduce the overall functional repertoire of a system, would be of particular interest.

\section{Acknowledgements}

Authors MS and RG were supported by the European Research Council under the European Union's Seventh Framework Programme (ERC-2010-AdG, ERC grant agreement n॰269853). Author GD was supported by the ERC Advanced Grant: DYSTRUCTURE. 
Author MPvdH was supported by a VENI grant of the Netherlands Organization for Scientific Research (NWO) and by a Fellowship of the Brain Center Rudolf Magnus.

\section{References}

Baars, B. J. (2005). Global workspace theory of consciousness: toward a cognitive neuroscience of human experience. The Boundaries of Consciousness: Neurobiology and Neuropathology, 150, 45-53.

Barabási, A. L., \& Albert, R. (1999). Emergence of scaling in random networks. Science, 286(5439), 509-512.

Bassett, D. S., Wymbs, N. F., Rombach, M. P., Porter, M. A., Mucha, P. J., \& Grafton, S. T. (2012). Core-Periphery Organisation of Human Brain Dynamics. arXiv:1210.3555.

Braga, R. M., Sharp, D. J., Leeson, C., Wise, R. J., \& Leech, R. (2013). Echoes of the Brain within Default Mode, Association, and Heteromodal Cortices. The Journal of Neuroscience, 33(35), 14031-14039.

Bullmore, E., \& Sporns, O. (2012). The economy of brain network organization. Nature Reviews Neuroscience, 13(5), 336-349.

Breakspear, M., Jirsa, V., \& Deco, G. (2010). Computational models of the brain: From structure to function. NeuroImage, 52(3), 727-730.

Colizza, V., Flammini, A., Serrano, M. A., \& Vespignani, A. (2006). Detecting rich club ordering in complex networks. Nature physics, 2(2), 110-115.

Collin, G., Hulshoff Pol, H. E., Haijma, S. V., Cahn, W., Kahn, R. S., \& van den Heuvel, M. P. (2011). Impaired Cerebellar Functional Connectivity in Schizophrenia Patients and Their Healthy Siblings. Frontiers in Psychiatry, 2.

Collin, G., Kahn, R. S., Reus, M. A. de, Cahn, W., \& Heuvel, M. P. van den. (2013a). Impaired Rich Club Connectivity in Unaffected Siblings of Schizophrenia Patients. Schizophrenia Bulletin, sbt162. 
Collin, G., Sporns, O., Mandl, R. C., \& van den Heuvel, M. P. (2013b). Structural and Functional Aspects Relating to Cost and Benefit of Rich Club Organization in the Human Cerebral Cortex. Cerebral Cortex.

De Reus, M. A., \& van den Heuvel, M. P. (2013). Rich Club Organization and Intermodule Communication in the Cat Connectome. The Journal of Neuroscience, 33(32), 1292912939.

Deco, G., Jirsa, V. K., \& McIntosh, A. R. (2010). Emerging concepts for the dynamical organization of resting-state activity in the brain. Nature Reviews Neuroscience, 12(1), $43-56$.

Deco, G., Senden, M., \& Jirsa, V. (2012). How anatomy shapes dynamics: a semi-analytical study of the brain at rest by a simple spin model. Frontiers in Computational Neuroscience, 6, 68.

Friston, K. J., \& Dolan, R. J. (2010). Computational and dynamic models in neuroimaging. Neuroimage, 52(3), 752-765.

Gibbs, J. W. (1905). Elementare Grundlagen der statistischen Mechanik: entwickeit besonders im Hinblick auf eine rationelle Begründung der Thermodynamik. JA Barth.

Ghosh, A., Rho, Y., McIntosh, A. R., Kötter, R., \& Jirsa, V. K. (2008). Noise during rest enables the exploration of the brain's dynamic repertoire. PLoS Comput Biol, 4(10), e1000196.

Harriger, L., van den Heuvel, M. P., \& Sporns, O. (2012). Rich club organization of macaque cerebral cortex and its role in network communication. PloS one, 7(9), e46497.

Honey, C. J., Kötter, R., Breakspear, M., \& Sporns, O. (2007). Network structure of cerebral cortex shapes functional connectivity on multiple time scales. Proceedings of the National Academy of Sciences, 104(24), 10240 -10245. doi:10.1073/pnas.0701519104

Hopfield, J. J. (1982). Neural networks and physical systems with emergent collective computational abilities. Proceedings of the national academy of sciences, 79(8), $2554-2558$.

Landau, L. D., \& Lifs $\square$ ic, E. M. (2007). Course of theoretical physics. 5 (3 ed.). Oxford: Pergamon Press. 
Maslov, S., \& Sneppen, K. (2002). Specificity and Stability in Topology of Protein Networks. Science, 296(5569), $910-913$.

McAuley, J. J., Da Fontoura Costa, L., \& Caetano, T. S. (2007). Rich club phenomenon across complex network hierarchies. Applied Physics Letters, 91(8), 084103-084103.

Scannell, J. W., Burns, G., Hilgetag, C. C., O’Neil, M. A., \& Young, M. P. (1999). The connectional organization of the cortico-thalamic system of the cat. Cerebral Cortex, 9(3), 277-299.

Senden, M., Goebel, R., \& Deco, G. (2012). Structural connectivity allows for multithreading during rest: The structure of the cortex leads to efficient alternation between resting state exploratory behavior and default mode processing. NeuroImage, 60(4), 2274-2284. doi:10.1016/j.neuroimage.2012.02.061

Towlson, E. K., Vértes, P. E., Ahnert, S. E., Schafer, W. R., \& Bullmore, E. T. (2013). The rich club of the C. elegans neuronal connectome. The Journal of Neuroscience, 33(15), 6380-6387.

Van den Heuvel, M. P., Kahn, R. S., Goñi, J., \& Sporns, O. (2012). High-cost, high-capacity backbone for global brain communication. Proceedings of the National Academy of Sciences, 109(28), 11372-11377.

Van den Heuvel, M. P., Stam, C. J., Kahn, R. S., \& Pol, H. E. H. (2009). Efficiency of Functional Brain Networks and Intellectual Performance. The Journal of Neuroscience, 29(23), 7619-7624.

Van den Heuvel, M. P., \& Sporns, O. (2011). Rich club organization of the human connectome. The Journal of Neuroscience, 31(44), 15775-15786.

Van den Heuvel, M. P., \& Sporns, O. (2013a). An Anatomical Substrate for Integration among Functional Networks in Human Cortex. The Journal of Neuroscience, 33(36), 14489-14500.

Van den Heuvel, M. P., \& Sporns, O. (2013b). Network hubs in the human brain. Trends in Cognitive Sciences, 17(12), 683-696. doi:10.1016/j.tics.2013.09.012

Watts, D. J., \& Strogatz, S. H. (1998). Collective dynamics of “small world”networks. Nature, 393(6684), 440-442.

Yu, Q., Sui, J., Liu, J., Plis, S. M., Kiehl, K. A., Pearlson, G., \& Calhoun, V. D. (2012). 
Disrupted correlation between low frequency power and connectivity strength of resting state brain networks in schizophrenia. Schizophrenia research. 143(1), 165 171.

Zamora-López, G., Zhou, C., \& Kurths, J. (2011). Exploring brain function from anatomical connectivity. Frontiers in neuroscience, 5.

Zhou, S., \& Mondragon, R. J. (2004). The rich club phenomenon in the Internet topology. IEEE Communications Letters, 8(3), 180 - 182. 


\section{Appendix: Metropolis Hastings Algorithm}

In what follows the Metropolis Hastings Algorithm used to estimate the entropy in a spin glass neural network is described. First, an initial global configuration was chosen by randomly selecting the number of spins that would initially be in an 'up' state from a normal distribution whose mean was $50 \%$ of spins in an 'up' and 50\% in a 'down' state and whose standard deviation was $12.5 \%$. Subsequently, spins were randomly selected and switched to an 'up' state until the number of spins supposed to be 'up' was reached. From this initial condition the algorithm proceeded by randomly selecting one spin and flipping it. If the energy of a thus selected trial configuration was lower as compared to the original configuration, the trial configuration was accepted. If the energy of the trial configuration was higher as compared to the original configuration, the trial configuration was accepted only with a certain probability:

$P_{\text {accept }}=e^{-\varepsilon \delta \mathrm{H}}$

where $\delta \mathrm{H}$ is the difference between the energy of the trial configuration and the energy of the original configuration: $\delta \mathrm{H}=H^{t}-H^{o}$.

These described steps were repeated for one million iterations for matrices comprising $\mathrm{N}=30$ nodes and ten million iterations for matrices comprising $\mathrm{N}=68$ nodes. In order to avoid the algorithm from getting stuck in local minima, occasionally (every 500 iterations), all spins were concurrently flipped such that every spin in an 'up' state switched to a 'down' state and vice versa. After the algorithm was finished, all unique global configurations that had been visited were retained. The entropy was calculated as described in the method section but only over the set of unique global configurations as obtained from running the algorithm. 


\section{Figure Captions}

Figure 1: Network architectures. The figure gives an illustrative representation of the artificial network architectures studied here. The upper row shows a regular (named the REG class, left), small world (SW, middle), and random (RAND, right) network. The lower row shows three scale free networks with different rich club organizations. Left is a typical scale free network without rich club organization (SF class). In the middle is the scale free network showing rich club organization (named the SF-RC class). Right is a scale free network showing negative rich organization (SF-negRC class). High degree hub nodes and hub-to-hub connections are shown in red.

Figure 2: Normalized rich club coefficient. The figure shows the normalized rich club coefficient given as a function of degree for the three types of scale free networks. As expected from the manipulation procedures, SF-RC networks exhibit a mean rich club coefficient exceeding 1 in the interval [4,7] (dark blue line). Normal SF networks in which hubs do not form a rich club exhibit a mean rich club coefficient around 1 (blue line); SFnegRC networks whose high-degree nodes show low levels of interconnectivity, exhibit a mean rich club coefficient consistently smaller than 1 (light blue line).

Figure 3: Entropy of the attractors of spin glass networks (24 nodes). The figure shows the entropy observed for each network class as a function of global coupling strength. All networks included the same network size and connectivity density (twenty-four nodes and 96 edges). The scale free networks show larger entropy levels over attractors than the corresponding small world (SW, yellow), regular (REG, red) and random (RAND, orange) networks. Additionally, within the category of scale free networks, the class of networks exhibiting rich club organization (SF-RC networks, dark blue) display the largest levels of 
entropy, while those exhibiting negative rich club organization (SF-negRC, light blue) show the lowest levels of entropy. Scale free networks without a rich club (SF, blue) show an intermediate level of entropy.

Figure 4: Marginal probability of 'up' states. The figure shows the marginal probability of being in an 'up' state for each node of a network architecture. The nodes are sorted by their degree. For all networks the marginal probability rises with degree. For random networks (orange) the rise resembles a logarithmic curve. In contrast, for scale free network architectures the marginal probability curve resembles an ' $\mathrm{S}$ ' curve with regions of low and high probability separated by a sharp rise. For SF-RC networks (dark blue) the region of high probability is smaller as compared to the other scale free type architectures. Additionally, for SF-RC networks two regions exist where nodes exhibit a lower probability of being active as compared to SF (blue) and SF-negRC (light blue) networks. These findings indicate that for scale free network architectures high-degree nodes contribute to every functional network while more low-degree nodes differentially contribute to different functional networks. For SF-RC networks more nodes were free to contribute to several functional repertoires.

Figure 5: Degree-related connectivity in artificial network architectures. The figure shows degree related connectivity for structural and functional connectivity derived from simulations of the three classes of scale free network architectures. For ease of interpretation the level of degree was divided into three categories, being low $(k=[2,5])$, moderate $(k=[6,10]$ and high $(\mathrm{k}=[11,14])$. (A) The first column shows average structural degree-related connectivity (DRC) for all types of network architectures. For SF networks (top row) connections between moderate and high-degree nodes are most prominent. As expected in the absence of a rich club, high-to-high connections are rare. For SF-RC networks (middle row) moderate-to-high connections are again most prominent, but in contrast to SF networks, 
networks with a SF-RC architecture feature a significant number of high-to-high connections. For SF-negRC networks (lower row) moderate-to-moderate connections are most prominent. Finally, in-degree connections among the most prominent nodes, namely those exhibiting low degree ( $76 \%$ of the nodes in the networks, see also supplementary figure 4 ), are largely absent for all three scale free network architectures. (B) The second column shows functional DRC for all scale free network architectures. For SF networks (upper row) moderate-to-moderate connections are most common, but they also feature some low-to-low and low-to-moderate connections. For SF-RC networks (middle row) low-to-low interactions are most common. For SF-negRC networks (lower row) the main concentration of connections is centralized among nodes of moderate degree. (C) The third column shows Euclidean distances between the centers of mass of functional and structural DRCs. The distribution was obtained via a bootstrapping procedure. This gives a clear indication where functional connections occur in the absence of underlying structural connections. That is, where the center of mass for structural DRC does not coincide with the center of mass for functional DRC. For SF and SFnegRC networks (upper and lower row, respectively) the distances are comparatively low. For SF-RC networks (middle row) the distances are comparatively large (several degrees).

Figure 6: Entropy of the attractors of spin glass networks (68 nodes). The figure shows the entropy observed for each network class (including human anatomy) as a function of global coupling strength. Human rewired (HUM-RW, blue) network architectures which retain the hub nodes present in human anatomy show larger entropy levels over attractors than the corresponding small world (SW, yellow), regular (REG, red) and random (RAND, orange) networks. More importantly, however, the human (HUM, dark blue) network architecture which additionally features a rich club displays the largest levels of entropy. 
Figure 7: Degree-related connectivity in empirical human connectivity data. The figure displays degree related connectivity for empirically derived human structural and functional connectivity. Similar to the artificial networks (figure 5), the level of anatomical degree was divided into the categories low $(\mathrm{k}=[4,10])$, moderate $(\mathrm{k}=[11,17]$ and high $(\mathrm{k}=[18,24]) .(\mathrm{A})$ The left panel shows human structural DRC, showing that moderate-to-moderate and moderate-to-high connections are most prominent. High-to-high connections exist, reflecting the rich club organization for the human connectome. (B) The right panel shows the functional DRC derived from empirical functional connectivity. Functional connections are present across all three categories with a strong cluster among low-degree nodes.

Supplementary Figure 1: Computation Time. The figure shows the time needed for solving the spin glass model for a single network as a function of its order (i.e. its number of nodes). The solid red line indicates the actual computation time if computations of states are run in serial. Computation time increases twofold with the addition of a node. The dashed red line indicates a projection of the computation time for networks of larger size if computations are run in serial. The black line indicates the computation time if computations of states are run in parallel using graphical processing units. Since simulations are performed for 100 matrices per type of network architecture and the parameter space for global coupling strength $\mathrm{W}$ is traversed in 41 steps the computation time needs to be multiplied by 4100 to arrive at the time needed to simulate a single architecture type.

Supplementary Figure 2: Normalized rich club coefficient (30 nodes). The figure shows the normalized rich club coefficient as a function of degree for the three types of scale free networks comprising 30 nodes. As observed for networks comprising 24 nodes, (figure 2, main text) SF-RC networks exhibit a mean rich club coefficient exceeding 1 in the interval [7,11] (dark blue line). SF networks whose hubs do not form a rich club exhibit a mean rich 
club coefficient around 1 (blue line); SF-negRC networks whose high-degree nodes show low levels of interconnectivity, exhibit a mean rich club coefficient consistently smaller than 1 (light blue line).

\section{Supplementary Figure 3: Entropy of the attractors of spin glass networks (30 nodes).}

The figure shows the entropy observed for each type of network architecture as a function of global coupling strength $W$. All networks contained thirty nodes and 180 edges. The left panel shows the semi-analytic solutions while the right panel shows approximations based on the application of the Metropolis-Hastings algorithm. In both cases, the scale free networks show larger entropy levels than the corresponding SW (yellow), REG (red) and RAND (orange) networks. Additionally, within the scale free class those exhibiting rich club organization (SFRC, dark blue) show the largest levels of entropy, while those exhibiting negative rich club organization (SF-negRC, light blue) show the lowest levels of entropy. Again highly consistent with the results of the $\mathrm{N}=24$, scale free networks without a rich club (SF, blue) show an intermediate level of entropy. The results of semi-analytic and approximate solutions are highly consistent and validate the use of the Metropolis-Hastings algorithm for estimation of relative effects with regard to global entropy in larger networks.

Supplementary Figure 4: Degree distribution for scale free network architectures. The figure shows the number of nodes exhibiting a certain degree $k$ over a range of degrees. As expected, low-degree nodes $(\mathrm{k}=[2,5])$ are most abundant $(76 \%)$ and high-degree nodes $(\mathrm{k}=[11,14])$ least abundant $(3 \%)$ for SF, SF-RC as well as SF-negRC. This overabundance of low-degree nodes implies that the diversity of the functional repertoire would greatly benefit if the low-degree nodes are functionally integrated. These effects are indeed observed in both scale free architectures containing a rich club and in empirical human networks (see main text and figures $5 \& 8$ ). 
Supplementary Figure 5: Degree distribution human data. The figure shows the number of nodes exhibiting a certain degree $k$ over a range of degrees. The body of the figure shows the distribution for the complete human connectivity data comprising 68 nodes. Low-degree nodes $(\mathrm{k}=[4,10])$ and moderate-degree nodes $(\mathrm{k}=[11,17])$ together form the majority, constituting $46 \%$ and $43 \%$ of all nodes in the network, respectively. High-degree nodes $(\mathrm{k}=[18,24])$ make up only a minority $(12 \%)$. This overabundance of low and moderate-degree nodes implies that the diversity of the functional repertoire would greatly benefit if these nodes are functionally integrated. These effects are indeed observed. The inlay shows the intra-hemispheric degree distribution for the left and right hemisphere (each 34 nodes) separately. Low-degree nodes $(\mathrm{k}=[4,8])$ and moderate-degree nodes $(\mathrm{k}=[9,13])$ together form the majority, constituting $51 \%$ and $40 \%$ of all nodes in the network, respectively. High-degree nodes $(\mathrm{k}=[14,18])$ make up only a minority $(8 \%)$. 


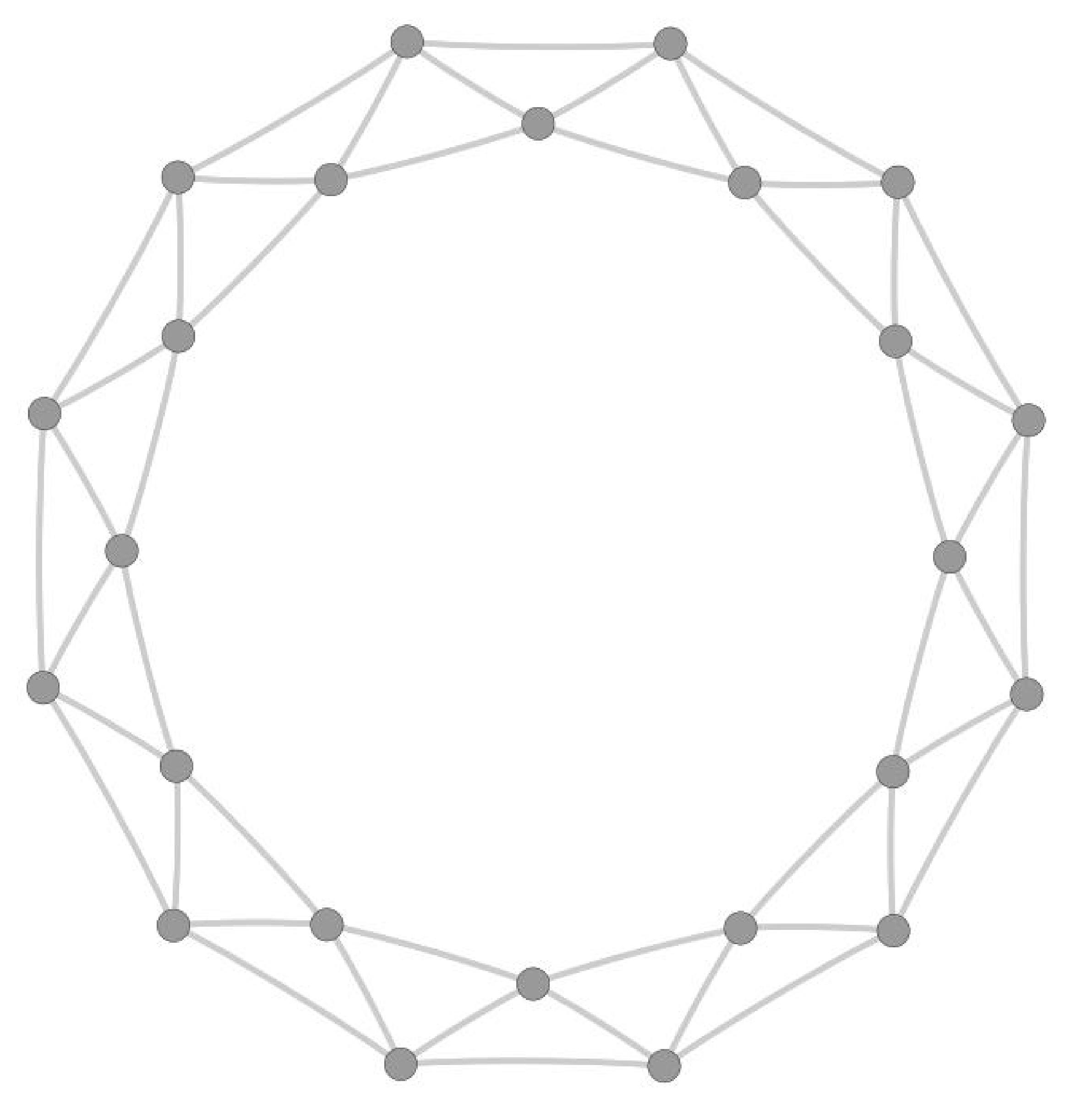

regular

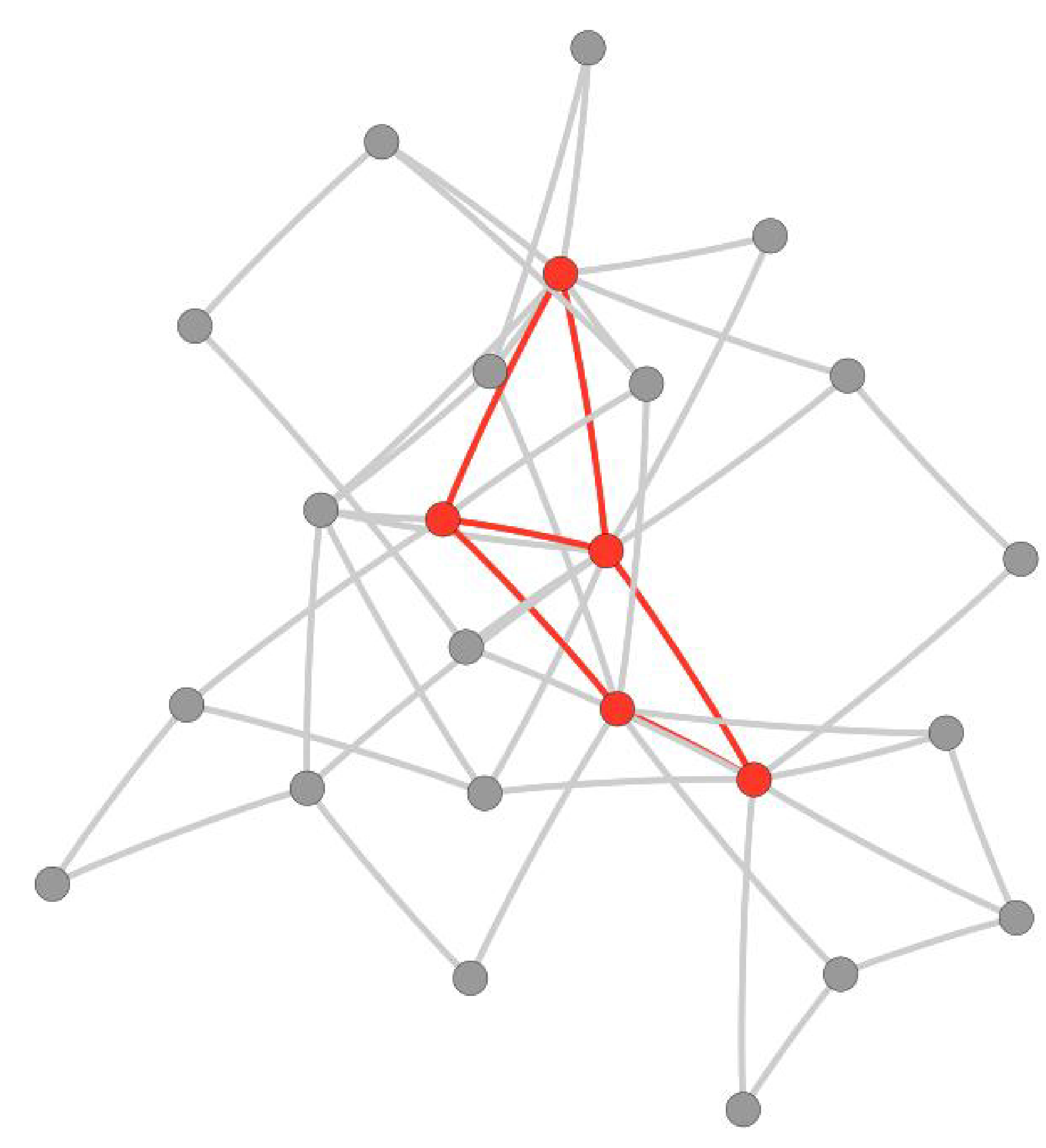

SF

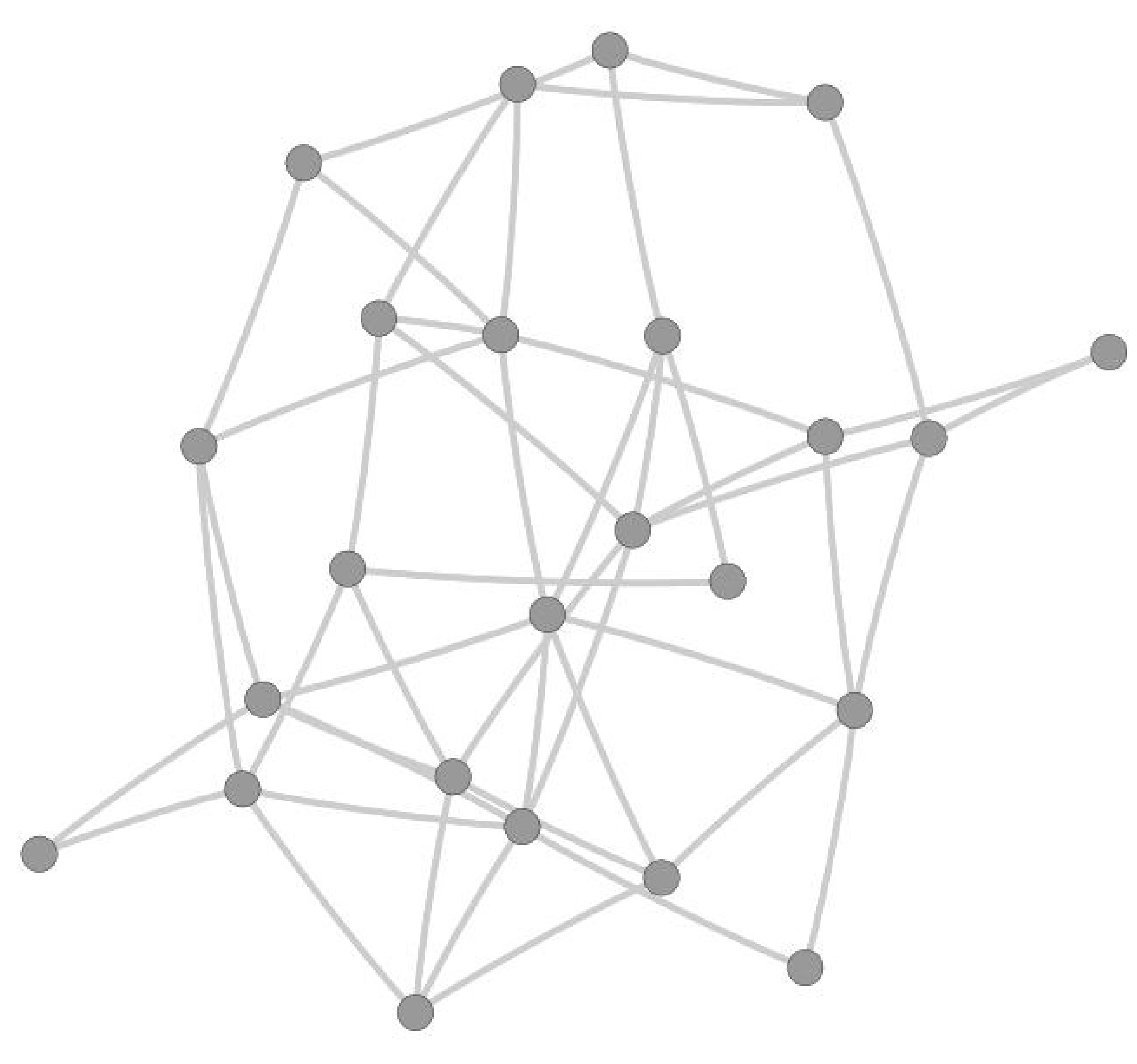

random

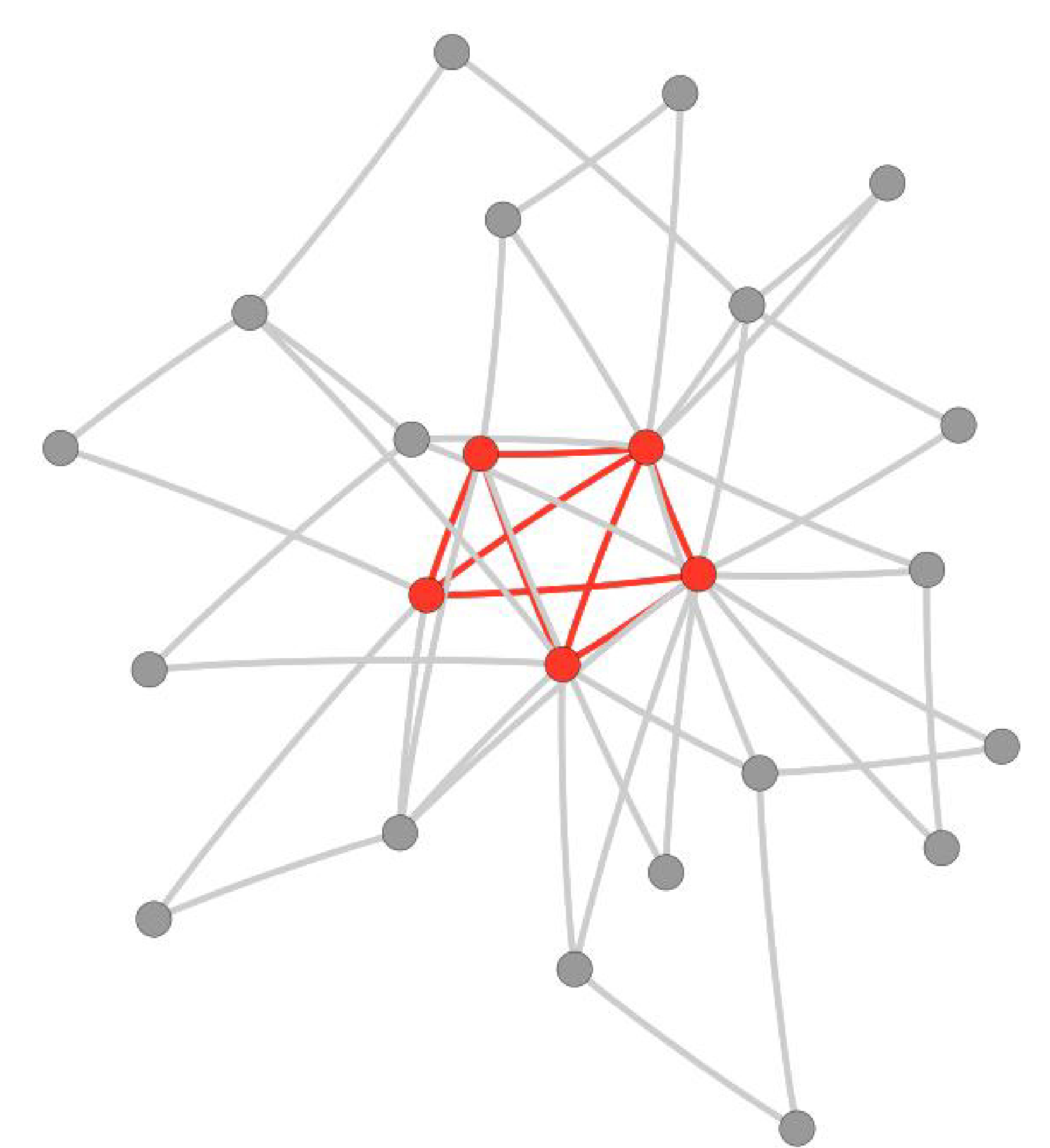

SF-RC

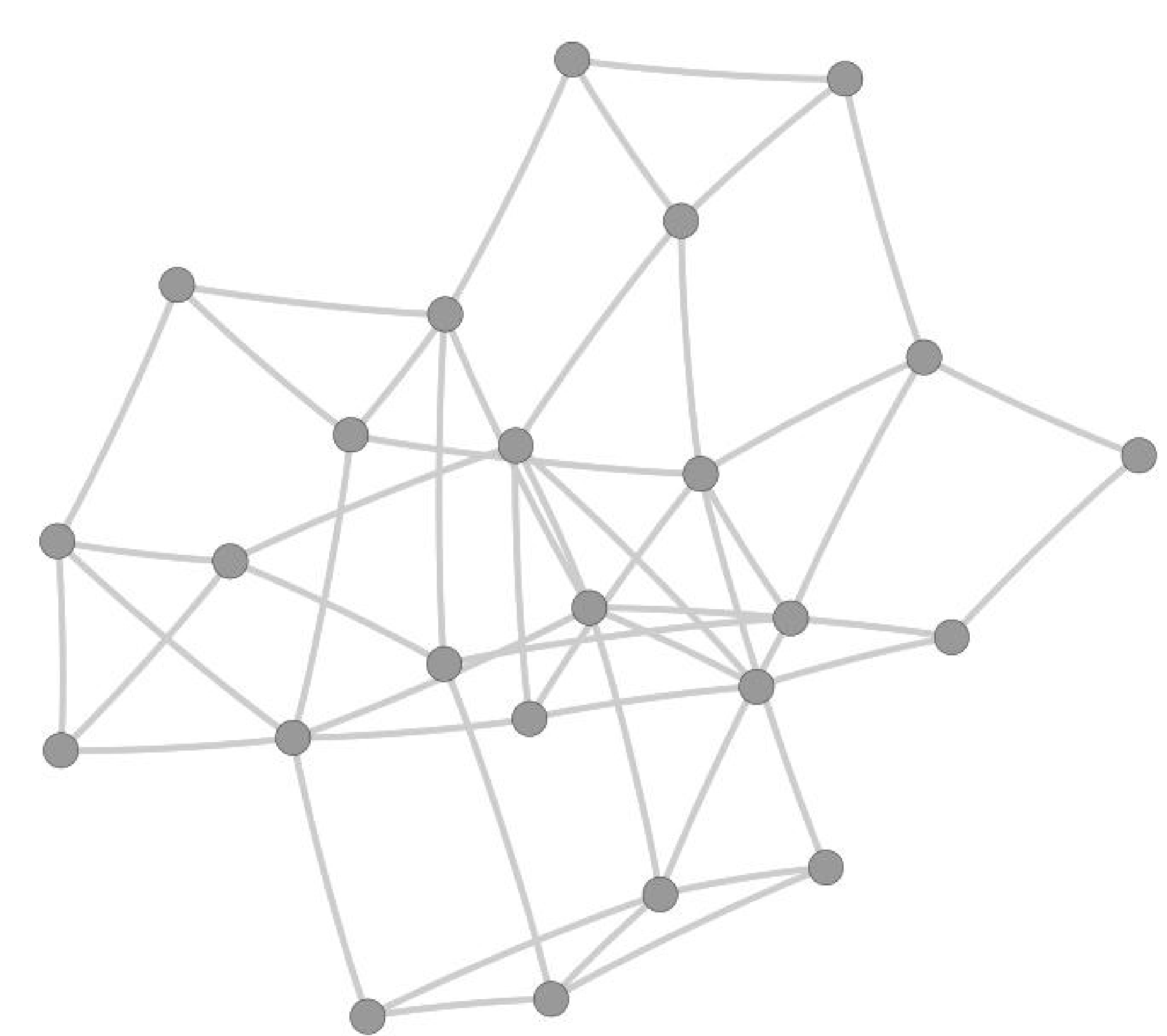

small-world

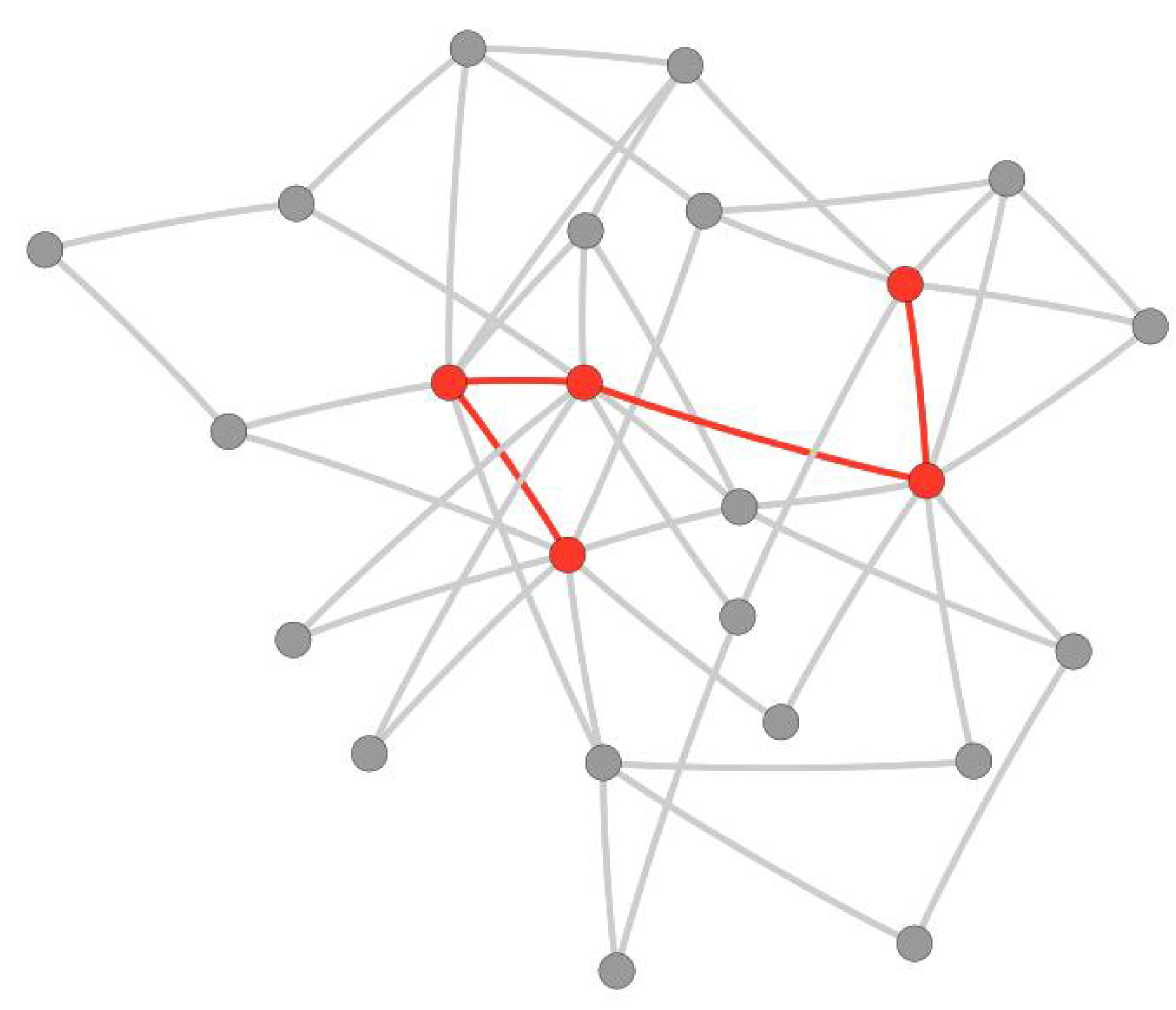

SF-negRC 
Click here to download high resolution image

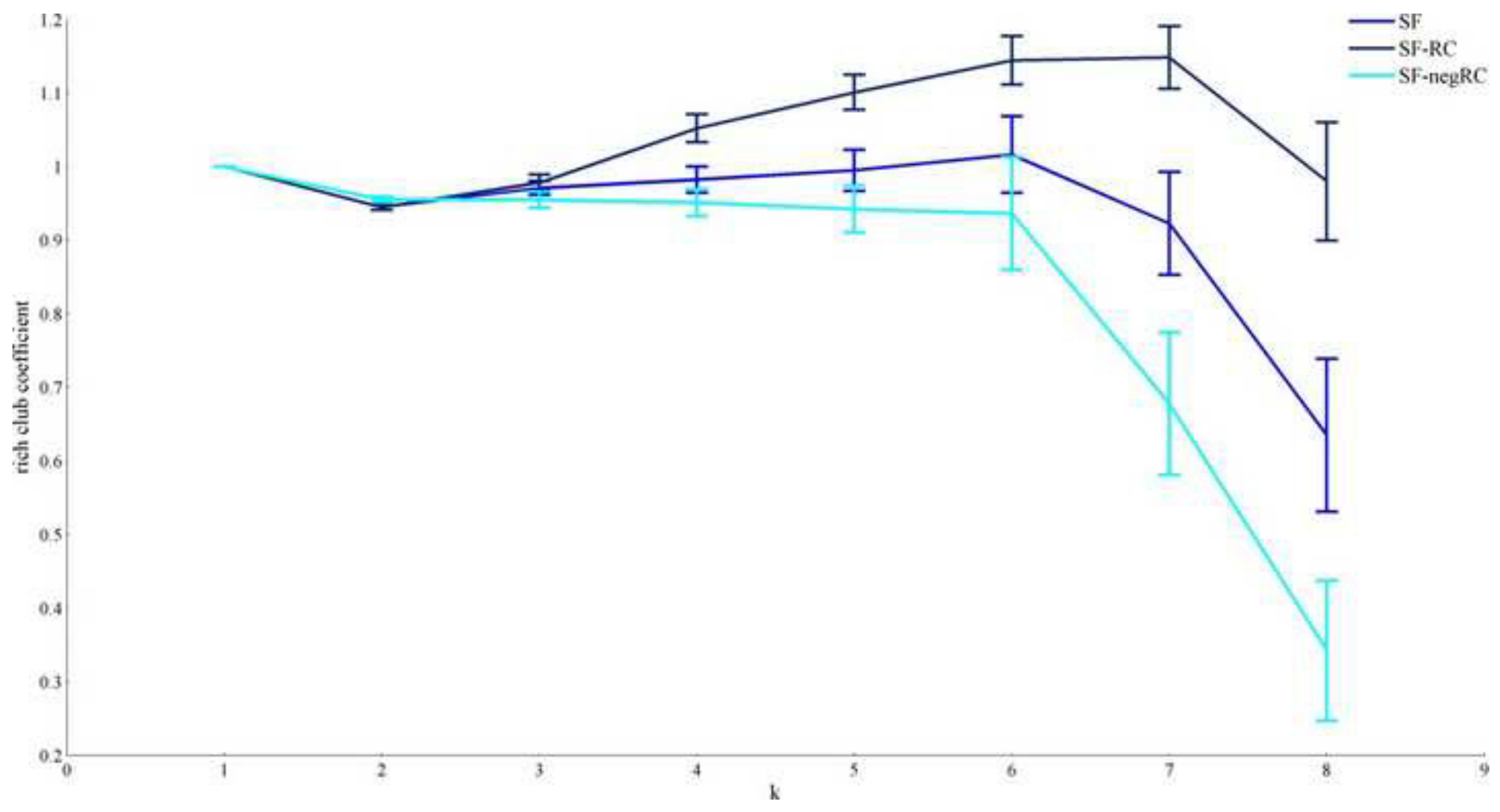


Click here to download high resolution image

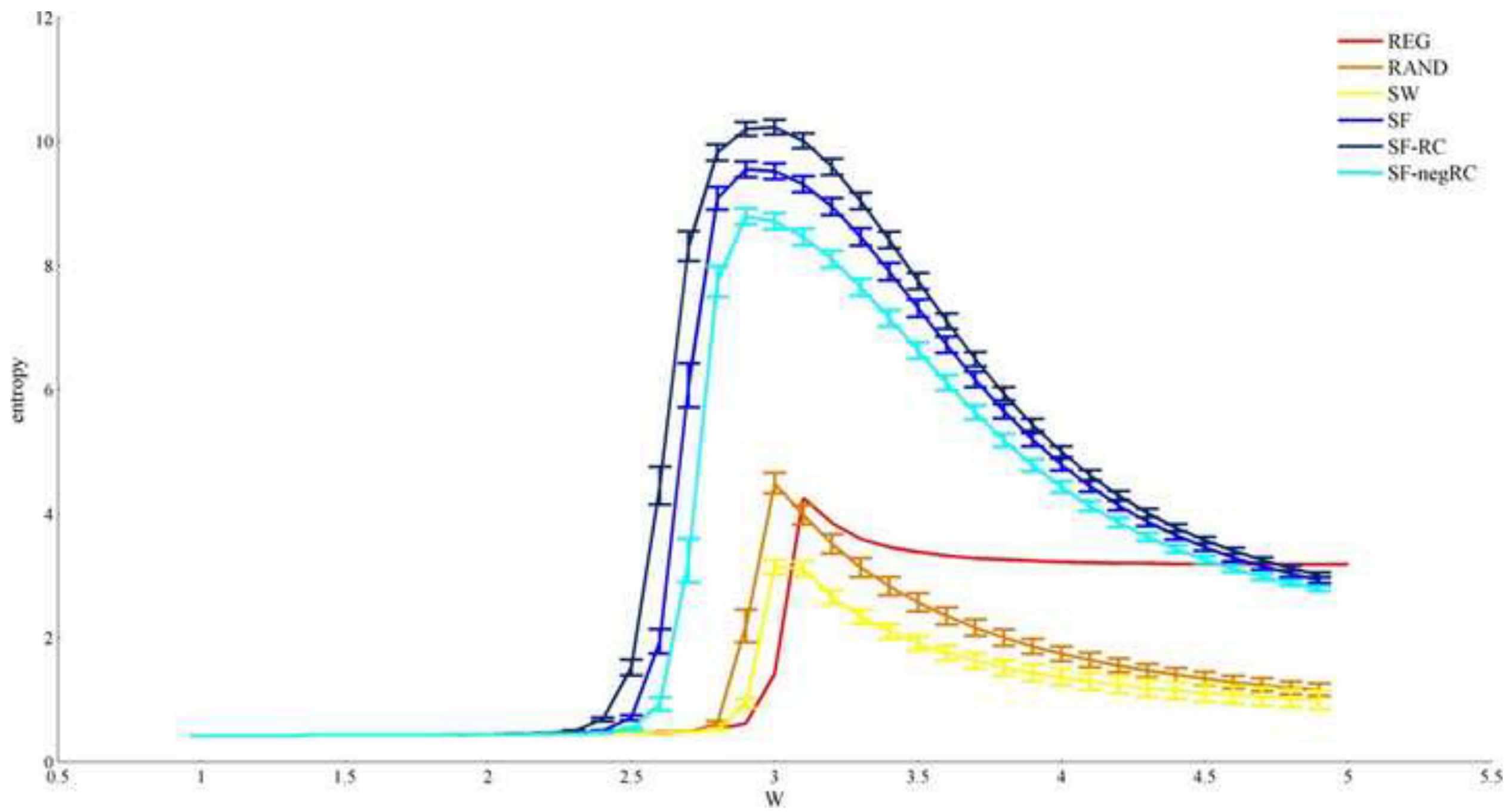




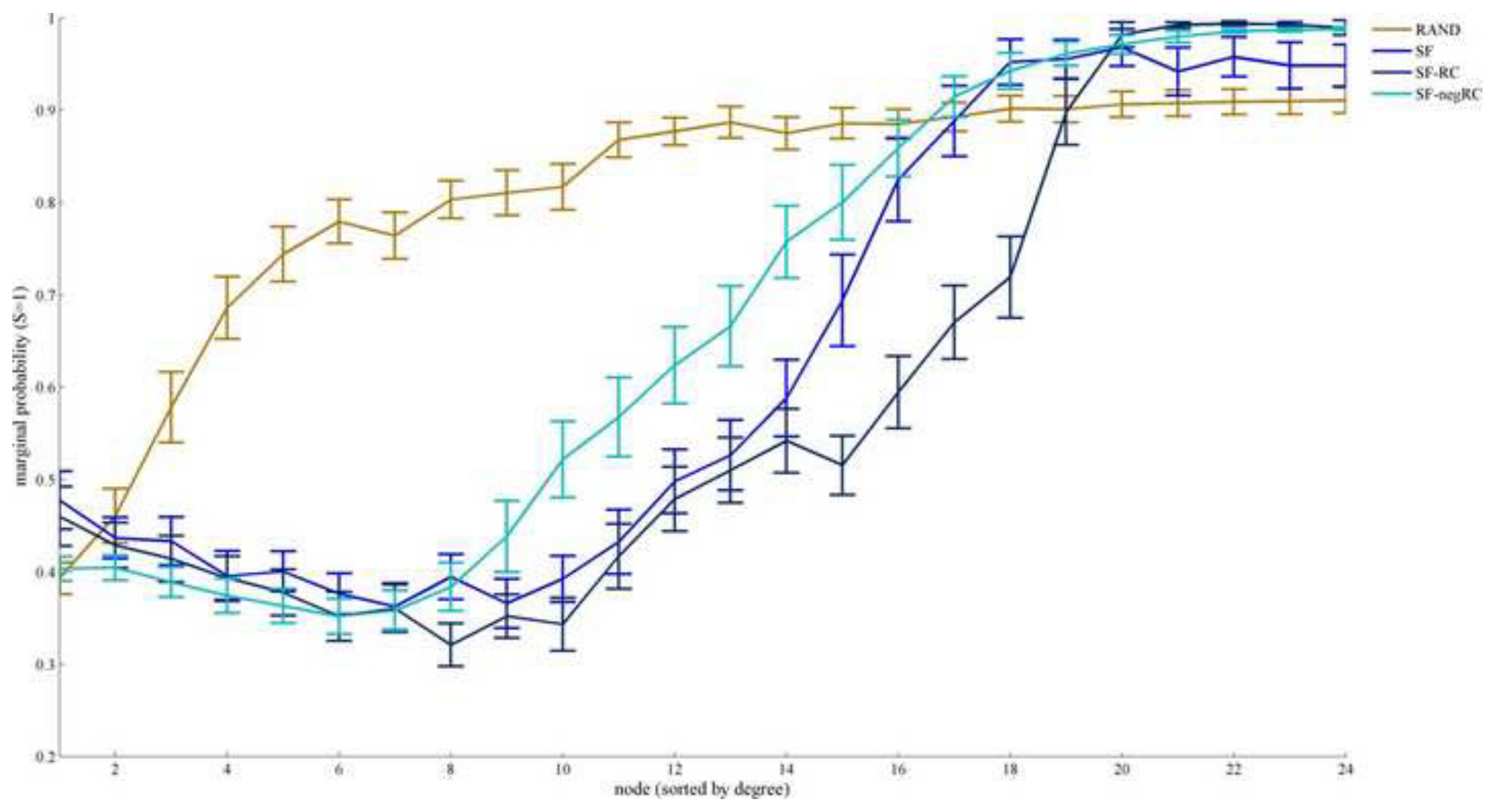


A
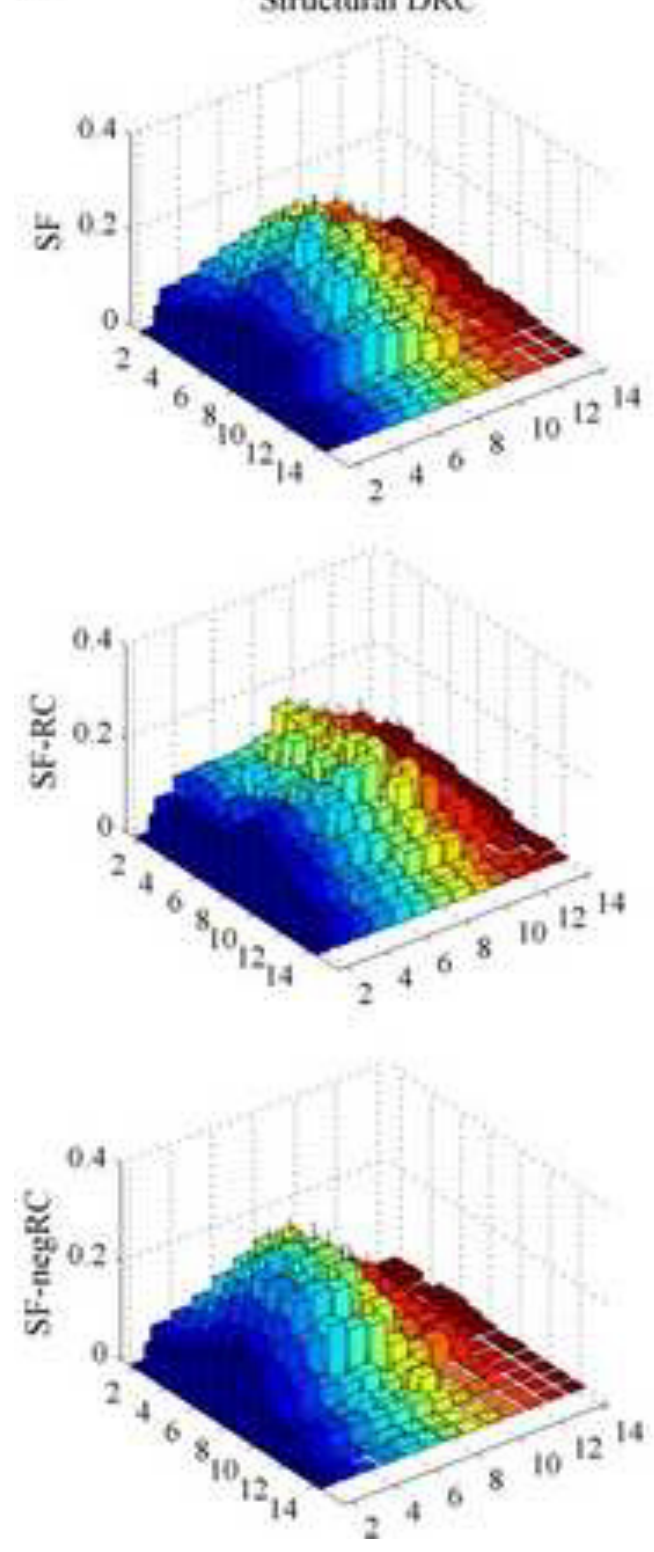

B
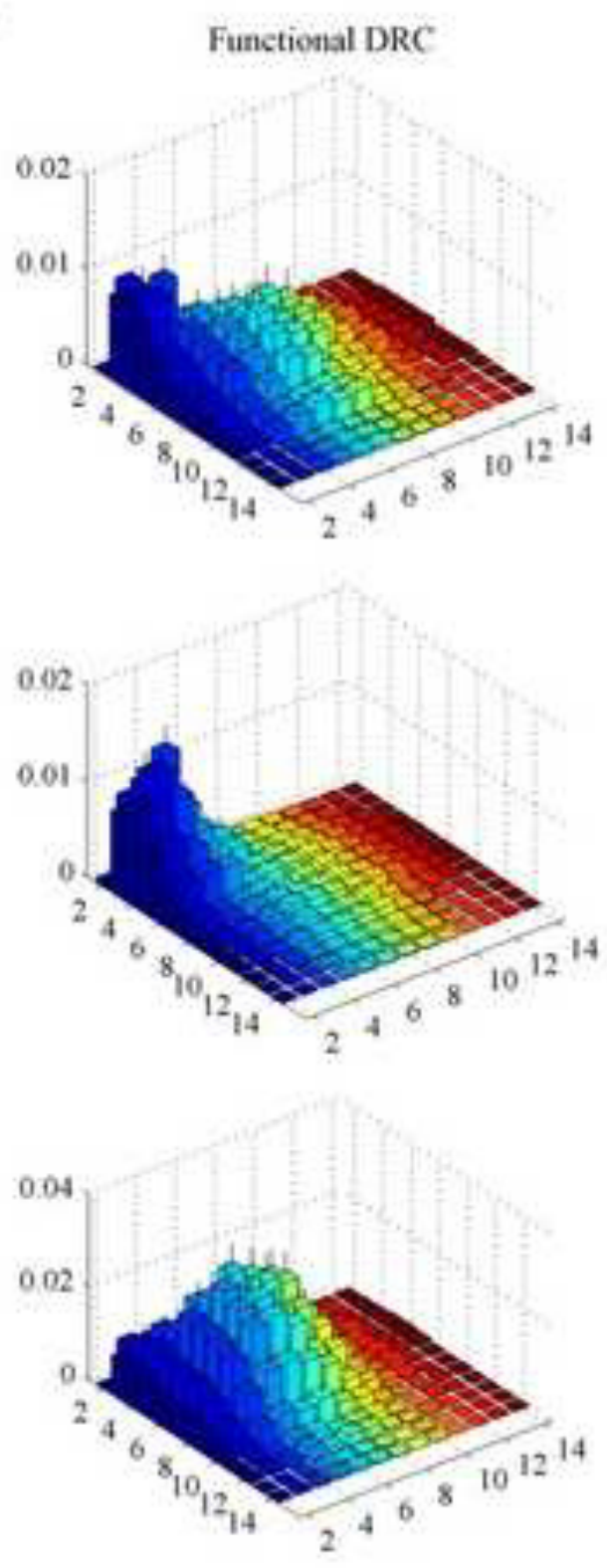

C
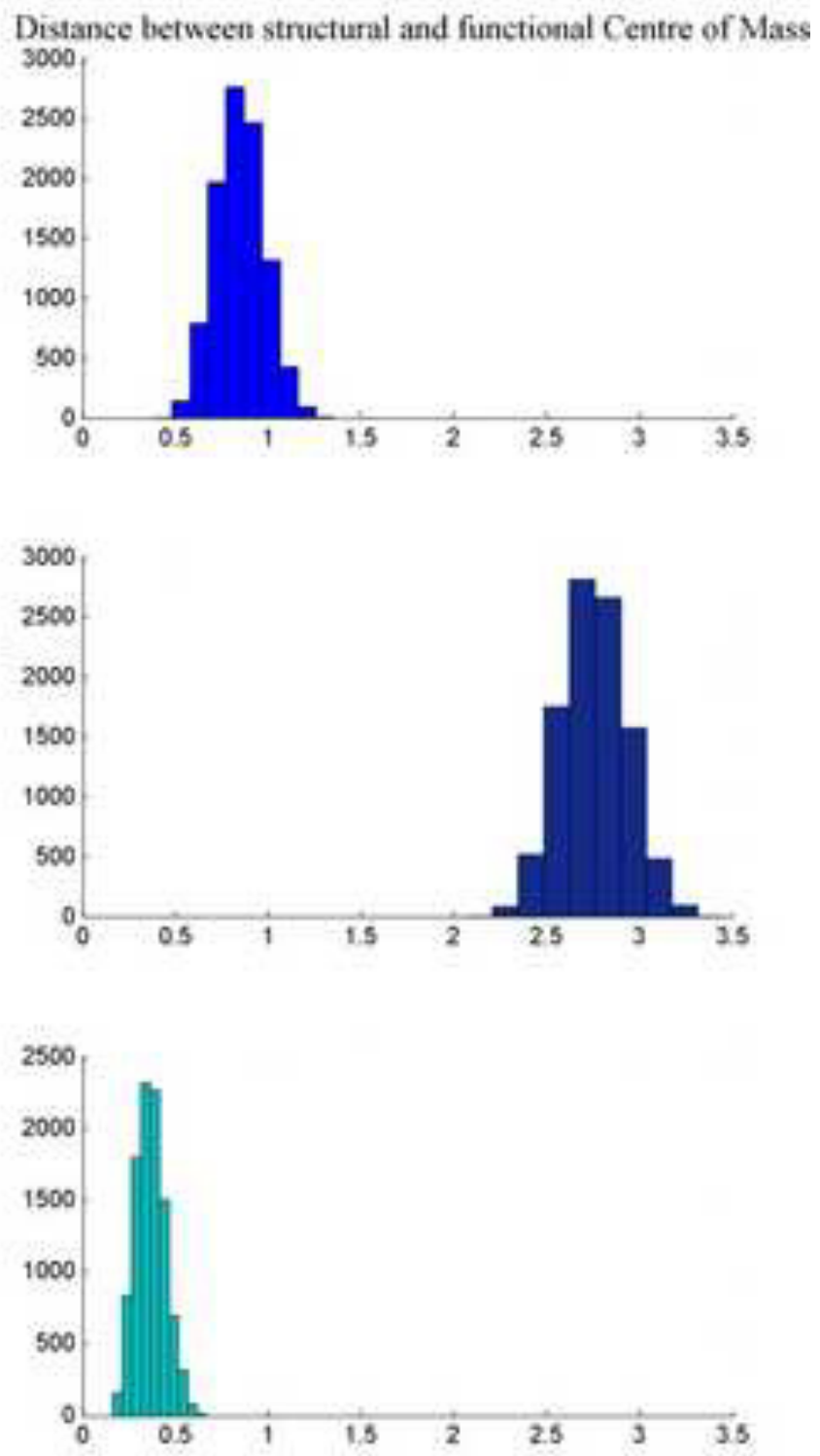
9. Figure

Click here to download high resolution image

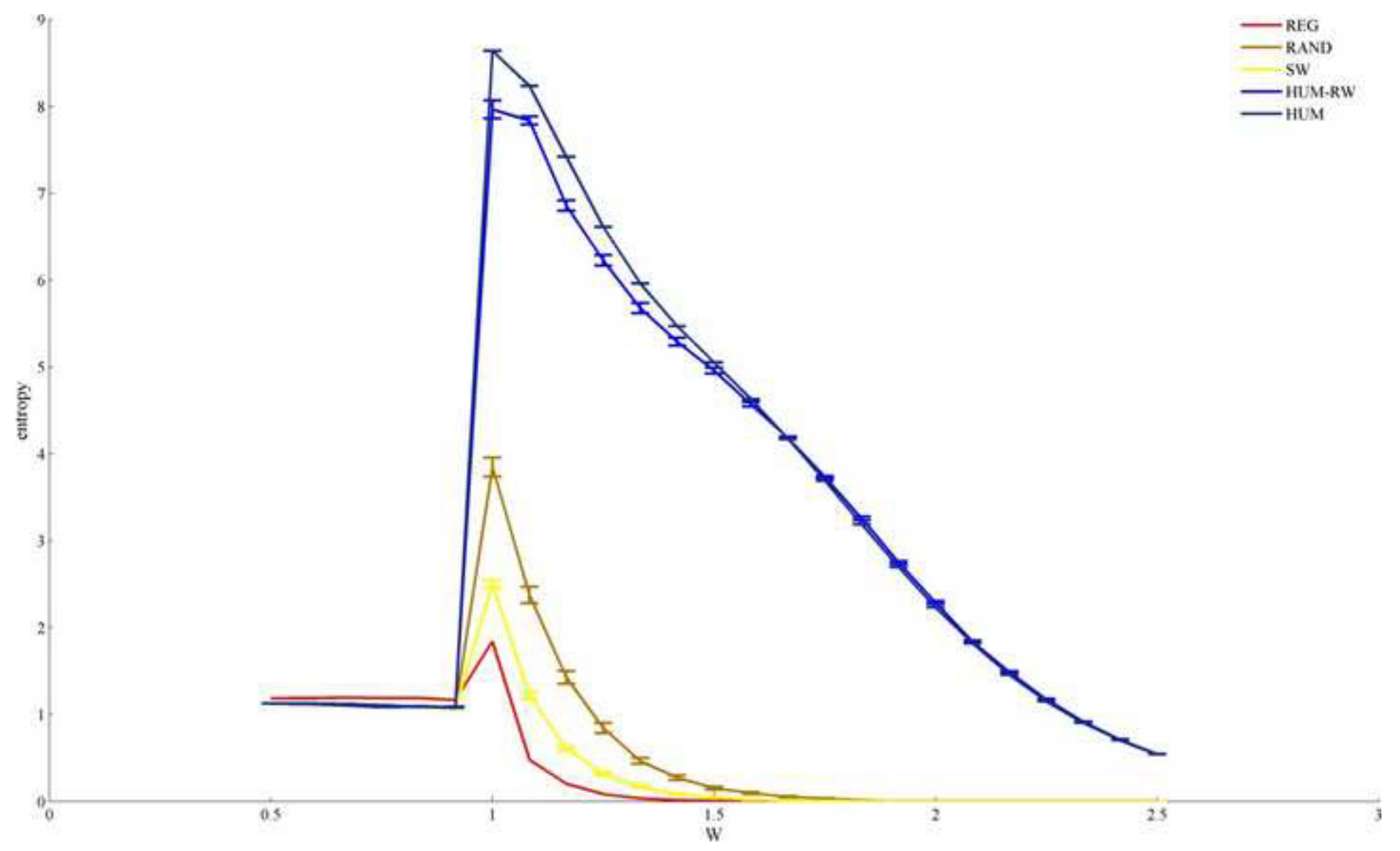




\section{Figure}

Click here to download high resolution image

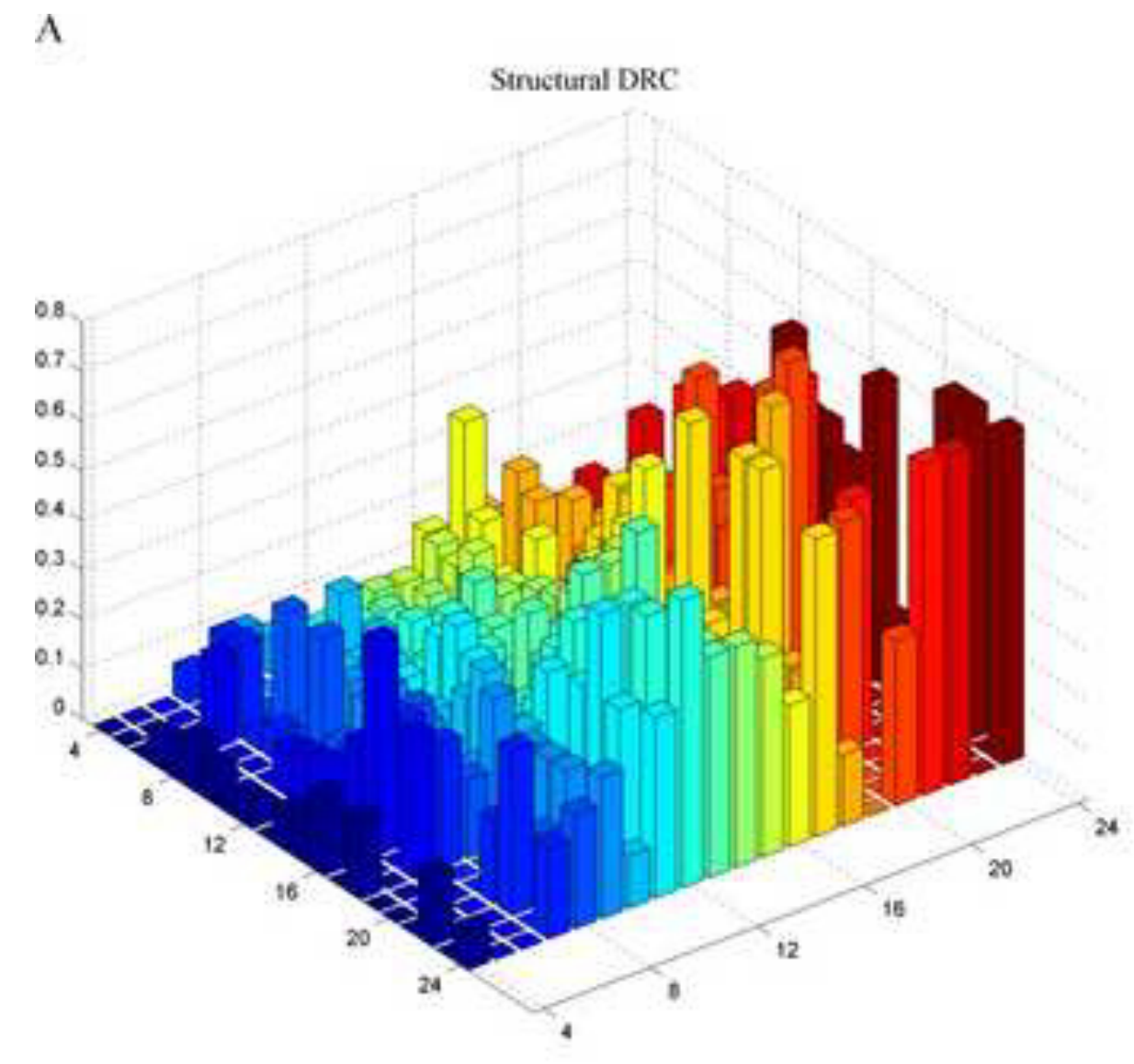

B

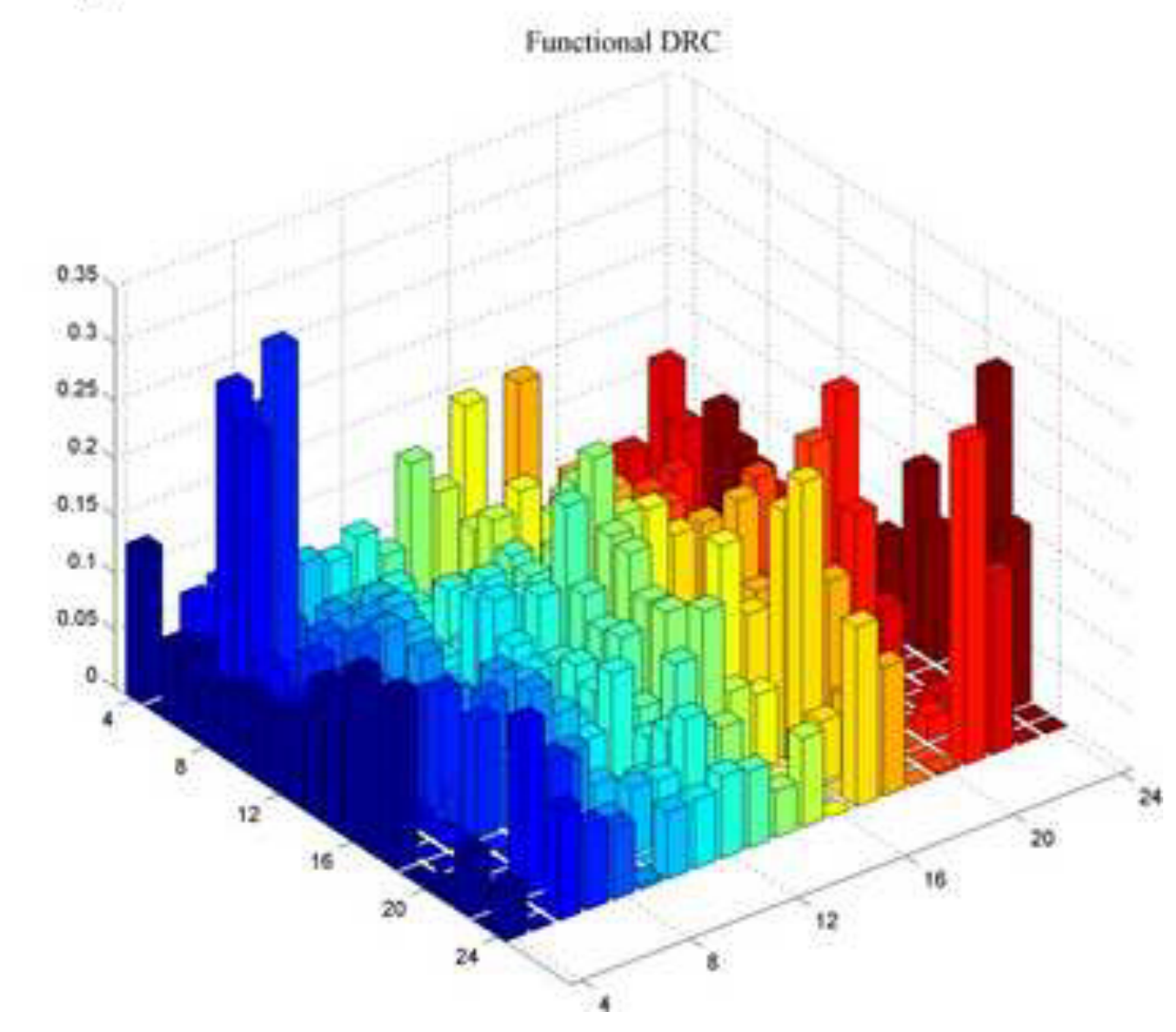




\section{Click here to download high resolution image}

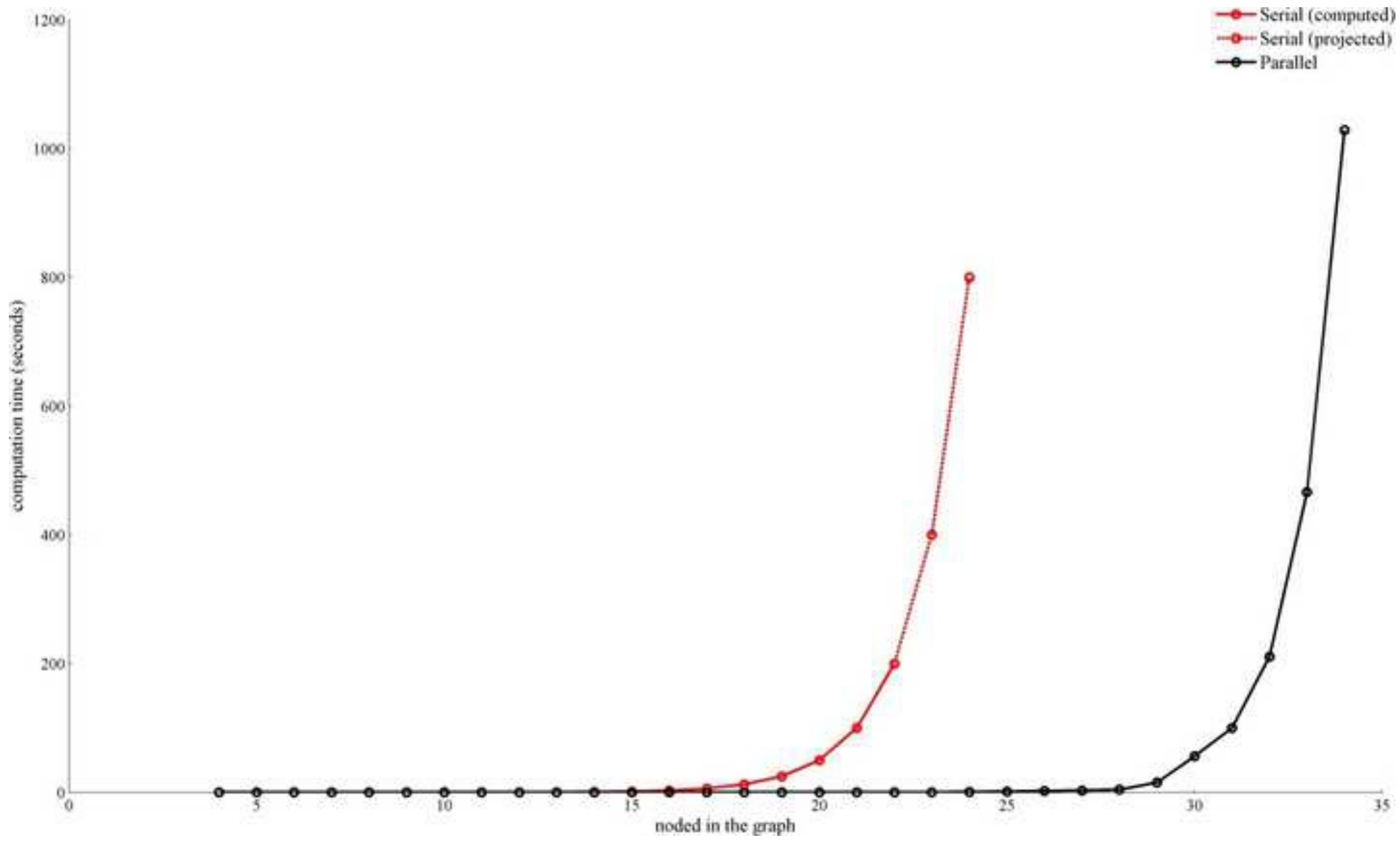


Click here to download high resolution image

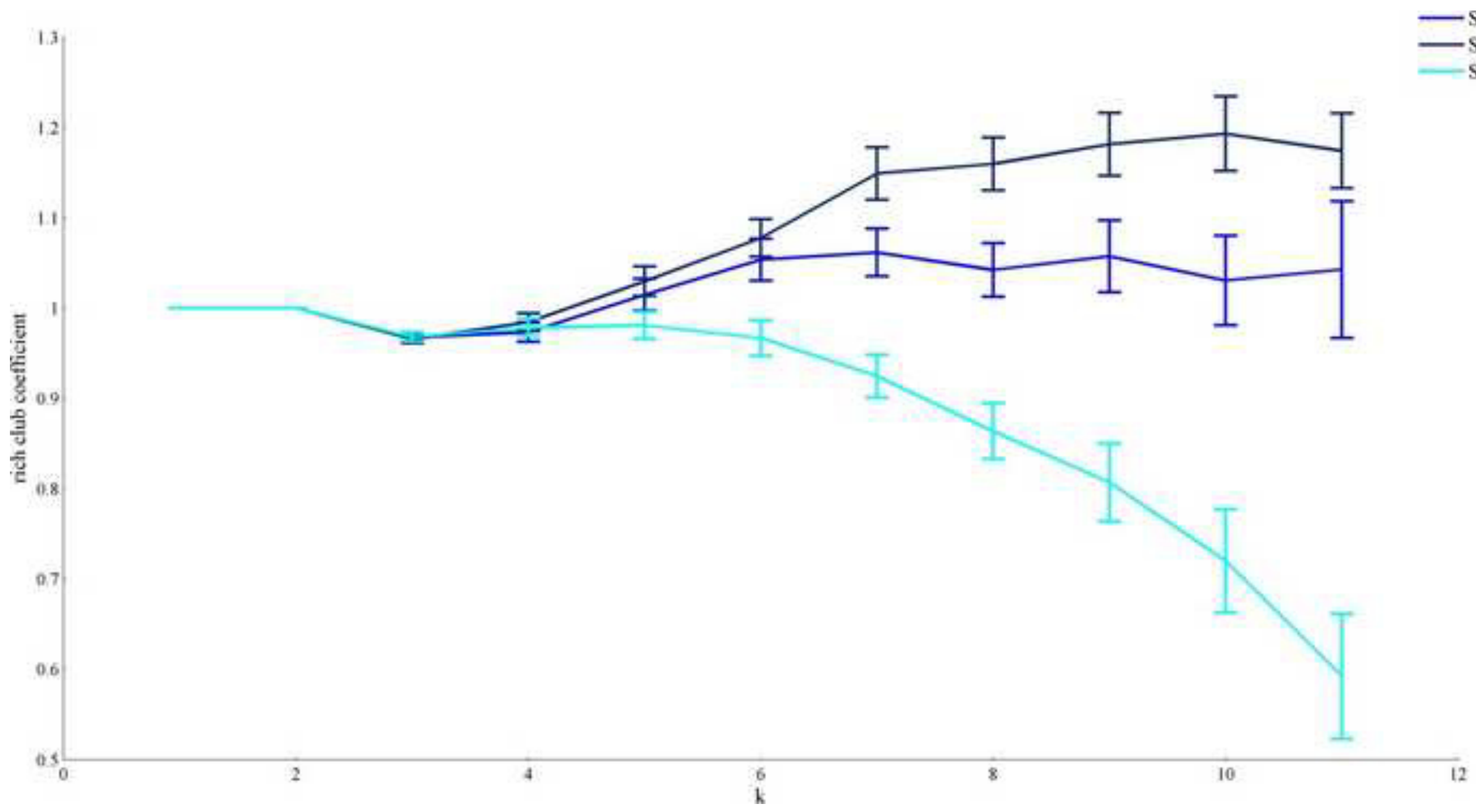



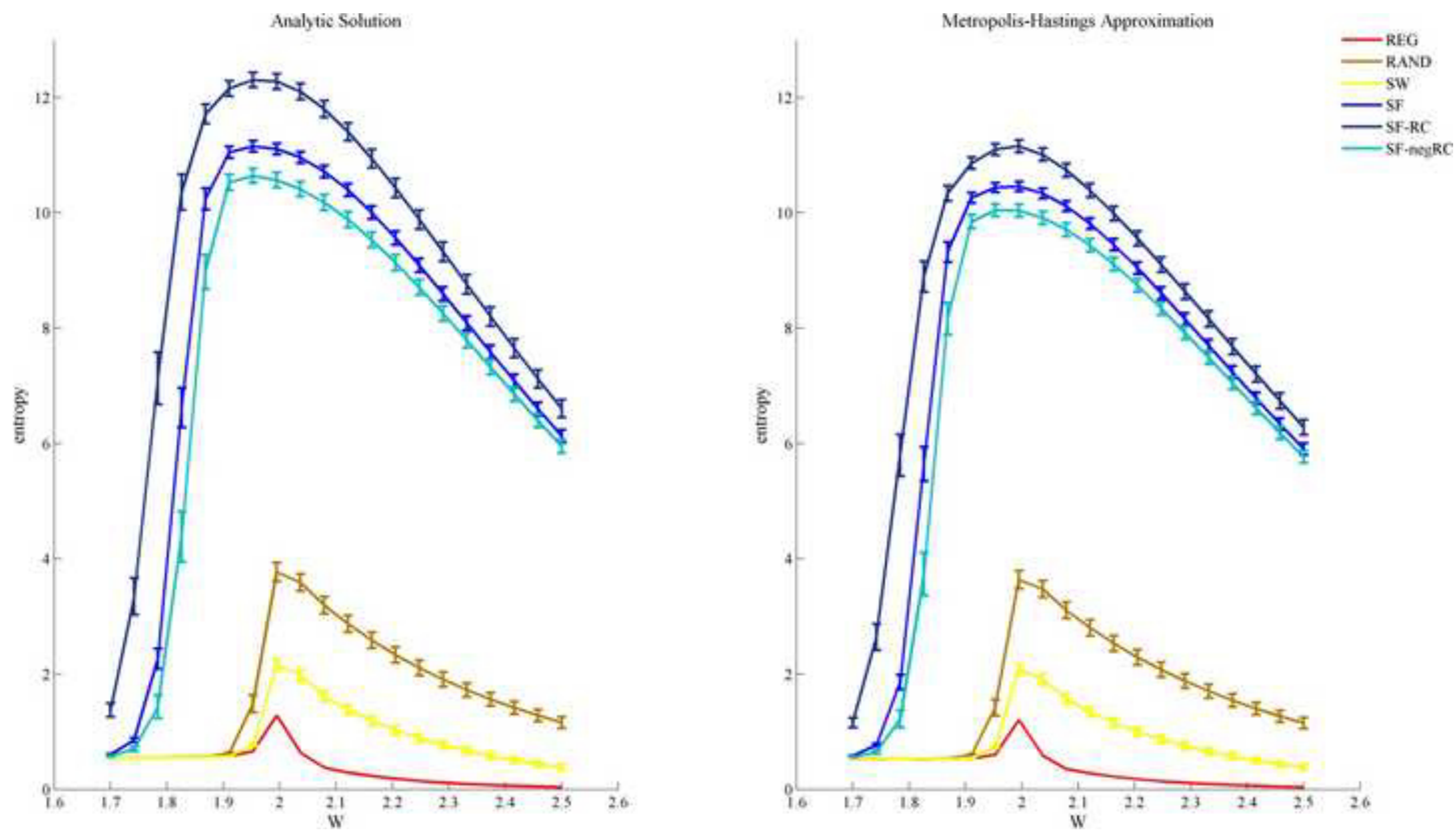
Click here to download high resolution image

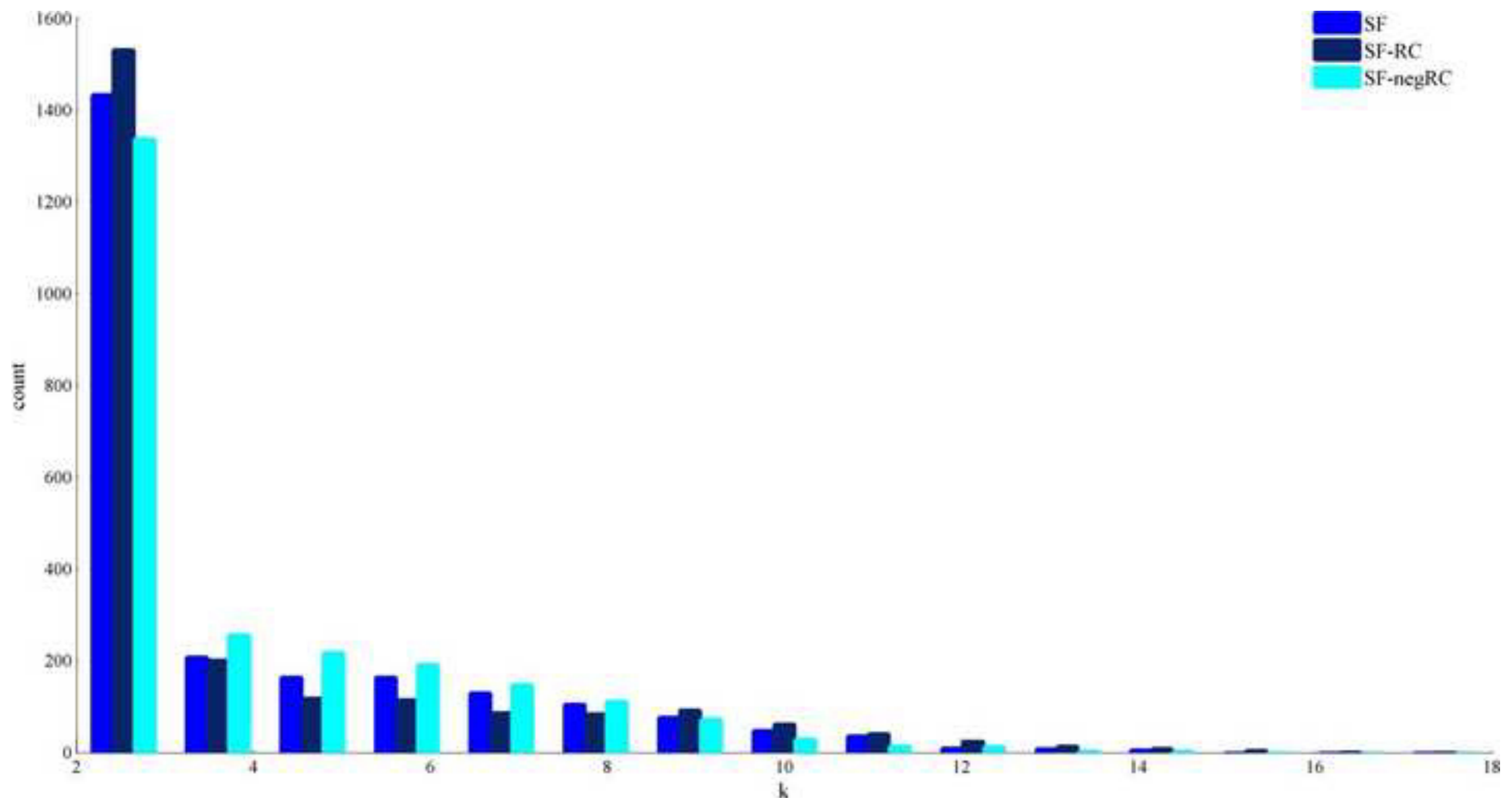




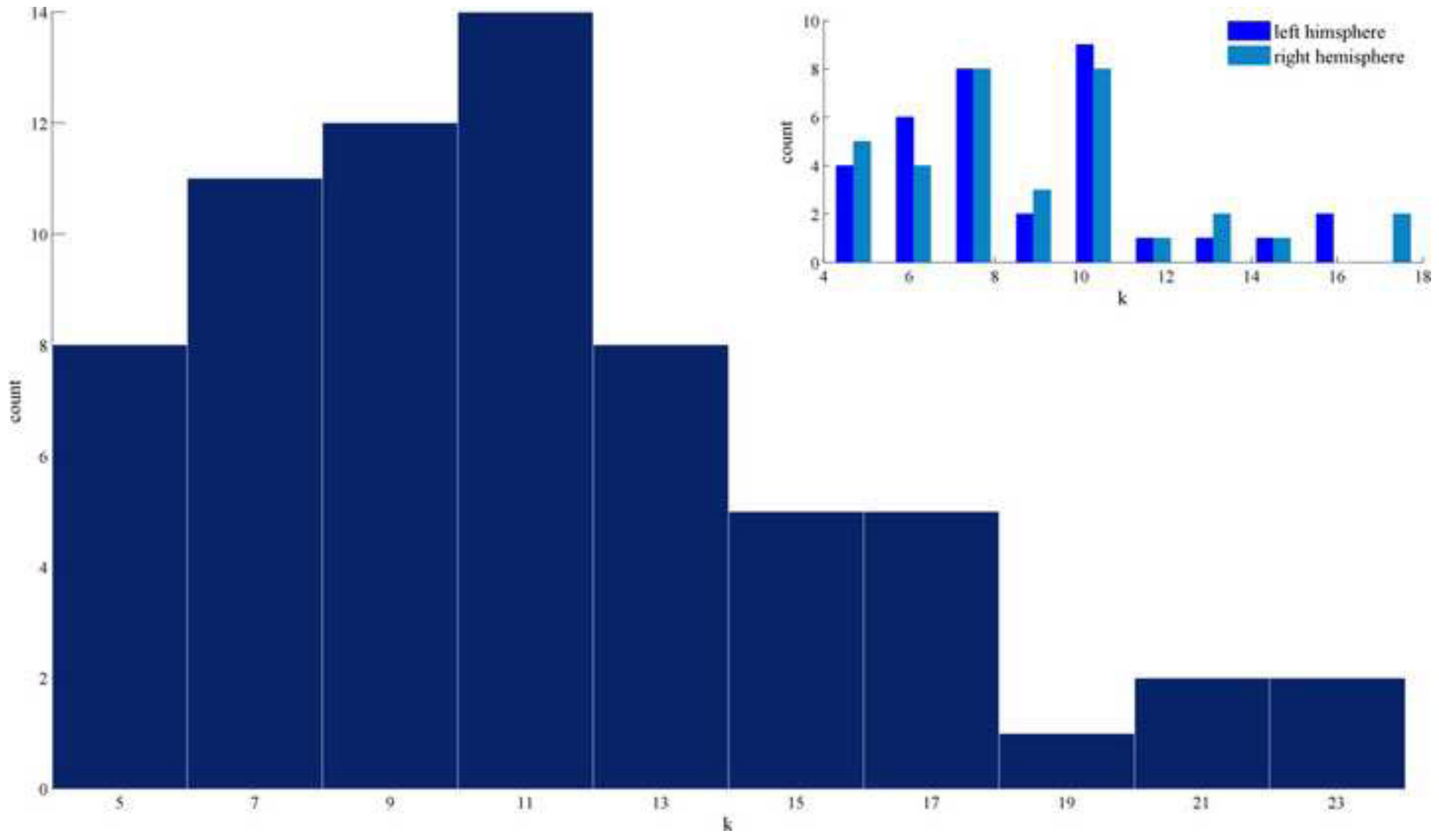

